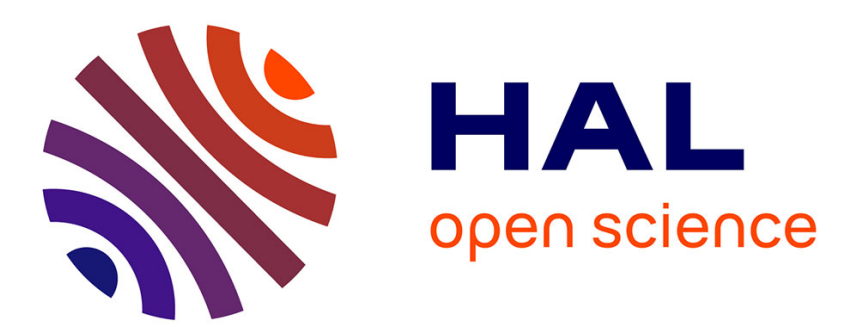

\title{
Galerkin method for solving combined radiative and conductive heat transfer
}

Mohamed Ghattassi, Jean Rodolphe Roche, Fatmir Asllanaj, Mohamed Boutayeb

\section{- To cite this version:}

Mohamed Ghattassi, Jean Rodolphe Roche, Fatmir Asllanaj, Mohamed Boutayeb. Galerkin method for solving combined radiative and conductive heat transfer. International Journal of Thermal Sciences, 2016, 102, pp.122-136. 10.1016/j.ijthermalsci.2015.10.011 . hal-01273062

\section{HAL Id: hal-01273062 \\ https://hal.science/hal-01273062}

Submitted on 19 Feb 2016

HAL is a multi-disciplinary open access archive for the deposit and dissemination of scientific research documents, whether they are published or not. The documents may come from teaching and research institutions in France or abroad, or from public or private research centers.
L'archive ouverte pluridisciplinaire HAL, est destinée au dépôt et à la diffusion de documents scientifiques de niveau recherche, publiés ou non, émanant des établissements d'enseignement et de recherche français ou étrangers, des laboratoires publics ou privés. 


\title{
Galerkin method for solving combined radiative and conductive heat transfer
}

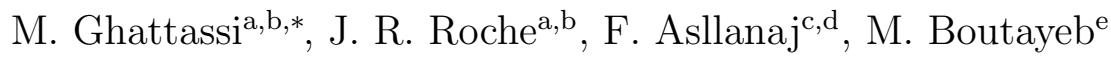 \\ ${ }^{a}$ Université de Lorraine, Institut Elie Cartan de Lorraine, UMR 7502, Vandoeuvre lès \\ Nancy, France \\ ${ }^{b}$ CNRS, Institut Elie Cartan de Lorraine, UMR 7502, Vandoeuvre lès Nancy, France. \\ ${ }^{c}$ Université de Lorraine, Laboratoire d'Energétique et de Mécanique Théorique et \\ Appliquée, UMR 7563, Vandoeuvre lès Nancy, France. \\ ${ }^{d}$ CNRS, Laboratoire d'Energétique et de Mécanique Théorique et Appliquée, UMR 7563 , \\ Vandoeuvre lès Nancy, France. \\ ${ }^{e}$ Université de Lorraine, Centre des Recherches en Automatique, UMR 7039, Vandoeuvre \\ lès Nancy, France.
}

\begin{abstract}
This article deals with a numerical solution for combined radiation and conduction heat transfer in a grey absorbing and emitting medium applied to a two-dimensional domain using triangular meshes. The radiative transfer equation was solved using the high order Discontinuous Galerkin method with an upwind numerical flux. The energy equation was discretized using a high order finite element method. Stability and error analysis were performed for the Discontinuous Galerkin method to solve radiative transfer equation. A new algorithm to solve the nonlinear radiative-conductive heat transfer systems was introduced and different types of boundary conditions were considered in numerical simulations. The proposed technique's high performance levels in terms of accuracy and stability are discussed in this paper with numerical examples given.
\end{abstract}

Keywords: Radiative transfer equation, Energy equation, Galerkin method, Newton Method, Error estimation.

\section{Nomenclature}

\footnotetext{
${ }^{*}$ Corresponding author

Email address: mohamed.ghattassi@univ-lorraine.fr (M. Ghattassi)
} 
$c_{p}$ specific heat capacity, $\mathrm{J} \mathrm{kg}^{-1} \mathrm{~K}^{-1}$

$\mathcal{C}^{0}(\Omega)$ continuous functions which vanish at infinity

$\mathcal{D}$ unit disk of $\mathbf{R}^{2}: \quad \mathcal{D}=\left\{\beta \in \mathbf{R}^{2}:|\beta| \leqslant 1\right\}$

$G^{\star}$ incident radiation intensity, $\mathrm{W} \mathrm{m} \mathrm{m}^{-2}$

$g$ dimensionless radiative boundary condition

$I^{\star}$ radiation intensity, $\mathrm{W} \mathrm{m}^{-2} \mathrm{sr}^{-1}$

$I_{b}$ blackbody intensity, $\mathrm{Wm}^{2} \mathrm{sr}^{-1}$

$h_{c}$ convective exchange coefficient at the boundary, $\mathrm{W} \mathrm{m}{ }^{-2} \mathrm{~K}^{-1}$

$k_{c}$ thermal conductivity of the medium, $\mathrm{W} \mathrm{m}{ }^{-1} \mathrm{~K}^{-1}$

$L$ thickness of the medium, $\mathrm{m}$

$n$ refractive index

n outward unit normal to the boundary

$N_{s}$ conduction radiation parameter

$B_{i}$ Biot number

$Q_{\text {rad }}$ radiative source term, $\mathrm{Wm}^{-2}$

$s^{\star}$ path length, $\mathrm{m}$

$s$ dimensionless path length

$S_{\text {rad }}^{\star}$ radiative source term, $\mathrm{Wm}^{-3}$

$t$ time, $\mathrm{s}$

$\mathcal{T}_{h}$ triangulation of $\Omega$

$T^{\star}, T_{0}^{\star}$ temperature, initial temperature in the medium, $\mathrm{K}$

$T, T_{0}$ dimensionless temperature, initial dimensionless temperature in the medium

$T_{\text {ref }}$ reference temperature, $\mathrm{K}$

\section{Greek symbols}

$\Omega$ dimensionless bounded domain in $\mathbf{R}^{2}$

$\Omega^{\star}$ bounded domain in $\mathbf{R}^{2}, \mathrm{~m}$

$\epsilon$ wall emission factor

$\kappa$ absorption coefficient, $\mathrm{m}^{-1}$

$\sigma_{B} \quad$ Stefan-Boltzmann constant

$\rho$ diffuse reflectivity or density, $\mathrm{kg} \mathrm{m}^{-3}$

$\beta$ direction of propagation of radiation

$\Delta t, \Delta \xi$ time step s, dimensionless step time

$\Gamma$ boundary of control volume

$\xi$ dimensionless time

$\theta=\frac{\kappa^{2} L^{2}}{N_{s}}$ dimensionless constant

Subscripts 
$l$ direction angular direction

$h$ step of the mesh

\section{Introduction}

The heat transfer models in participating media for scientific purpose or engineering applications such as high temperature processing or thermal insulation involve coupled radiation and conduction heat equations $[1,2,3$, $4,5,6]$. The most fundamental difficulties for the simulation of radiative and conductive heat transfer problems are the nonlinear coupling terms and evolutive boundary conditions. Historically, many simplified models have been used to carry out numerical simulations of radiative transfer phenomena. Generally, two classes of numerical methods have been applied to attempt to solve radiative heat transfer problems namely the Monte Carlo method $[7,8]$ and the deterministic methods $[9,10,11]$.

In this study, we chose the Discontinuous Galerkin (DG) method, a deterministic method, to solve the radiative transfer equation(RTE). The DG method is known to be locally conservative, stable and high-order accurate. It can also easily handle complex geometries, irregular meshes with hanging nodes and allows for different degrees of approximation for different elements[12, 13]. The DG method is known to be a particularly powerful numerical scheme for the simulation of hyperbolic transport problems. The nodal DG method can be easily combined with the well known finite element methods (FEM) or finite volume methods (FVM) to couple radiative and conductive heat transfer. Spatial DG techniques applied to discreteordinates radiation transport have been pioneered by Reed and Hill [14] and Lesaint and Raviart [15].

In [16], the DG method was extended to the solution of radiative heat transfer problems in absorbing, emitting and scattering media. In [16] the authors studies a centered numerical flux and implemented a parallel computing algorithm based around the localized DG formulation. In [17] a 3-d model was reduced to 2 -d using symmetry and the DG formulation. In $[18,19]$, the authors added a stabilization term to penalize the jump of the solution across interior faces of the triangulation and to obtain physical numerical solutions in practical applications. For the transient state, many authors have considered radiation and conduction coupled problem, see [20, 21, 22]. Mishra et al. [22], applied the lattice Boltzmann method to solve the energy equation of 
a transient conduction-radiation problem in a 2-d rectangular enclosure and the collapsed-dimension method was implemented to solve the RTE. Asllanaj et al. [20], simulated transient heat transfer by radiation and conduction in two-dimensional complex shaped domains with structured and unstructured triangular meshes working with an absorbing, emitting and non-scattering grey medium. To solve the RTE, these authors applied a modified FVM based on a cell vertex scheme combined with a modified exponential scheme where temperature was approximated by linear interpolation using nodal values. The PHAML (Parallel Hierarchical Adaptive Multi Level) code was used to solve the heat conduction equation in space, with low or high order finite elements.

The objective of the work covered in this paper was to solve the nonlinear system which describes the heat transfer in a grey absorbing and emitting medium. We introduced a high order DG method based on an upwind stable numerical flux to solve the radiative heat transfer problem and a high order finite element method to solve the energy equation. Indeed, the high order Galerkin method ensures a more accurate approach with a computational time gain. We can also generalize this method to solve combined radiation conduction heat transfer in irregular geometries. An adequate norm was introduced to prove the stability of DG method for solving RTE and we shall also give an adapted error estimate which we found to be accurate in our simulations. A new numerical algorithm to solve the considered nonlinear-coupled radiative-conductive problem is given in this paper along with numerical results for high order scheme. We investigated a new type of boundary conditions and obtained new numerical results for cases not covered in previous publications. Finally we shall show that numerical stability was verified in our simulations.

The outline of this paper is as follows. In the next section, the radiativeconductive heat transfer in the absorbing emitting medium will be briefly described and the dimensionless form is introduced. The radiative boundary condition and its corresponding mixed thermal boundary conditions will be presented. Discretization of the RTE and energy equation by the discrete DG method and the finite element method respectively, are discussed in detail in Section 3 and also established the stability of the DG method with the adequate norm. Finally, in Section 4, we shall investigate the coupled heat transfer problem and give results showing the effects of the conductionradiation parameter and wall emissivity on radiative and conductive heat fluxes. 


\section{Problem statement}

In this section, the radiative-conductive heat transfer system of partial differential equations in a two dimensional gray absorbing and emitting medium is introduced. We denote

$$
\partial \Omega_{-}^{\star}=\left\{\left(s^{\star}, \beta\right) \in \partial \Omega^{\star} \times \mathcal{D} \text { such that } \beta . \mathbf{n}<0\right\} .
$$

The unknown of the RTE is the radiation intensity denoted $I^{\star}\left(t, s^{\star}, \beta\right)$ given at time $t$, position $s^{\star}$ and in the direction $\beta$. The unknown of the energy equation is the temperature $T^{\star}\left(t, s^{\star}\right)$ at time $t$ and position $s^{\star}$. The RTE is given by (see,[20])

$$
\begin{array}{rlrl}
\beta . \nabla I^{\star}\left(t, s^{\star}, \beta\right)+\kappa I^{\star}\left(t, s^{\star}, \beta\right) & =\kappa n^{2} I_{b}\left(T^{\star}\left(t, s^{\star}\right)\right), & & \text { for }\left(t, s^{\star}, \beta\right) \\
I^{\star}\left(t, s^{\star}, \beta\right) & =g^{\star}\left(t, s^{\star}, \beta\right), \quad & \text { for } \left.\quad\left(t, s^{\star}, \beta\right) \in\left[0, \tau^{\star}\right] \times \tau^{\star}\right] \times \partial \Omega_{-}^{\star},
\end{array}
$$

where $\kappa$ is the absorption coefficient of the medium and $n$ is the refractive index. In this work, the refractive index and the absorption coefficient are assumed to be equal to one, $n=1, \kappa=1 m^{-1} . I_{b}\left(T^{\star}\right)$ is the radiation intensity of the blackbody with, $T^{\star}$, the temperature of the medium:

$$
I_{b}\left(T^{\star}\right)=\frac{\sigma_{B}}{\pi} T^{\star 4},
$$

where $\sigma_{B}=5.6698 \times 10^{-8} W^{-2} K^{-4}$ is the Stefan-Boltzmann constant. The radiative boundary condition $g^{\star}\left(t, s^{\star}, \beta\right)$ takes into account of two quantities, the emitted radiation intensity and the incoming radiation. For an opaque wall with specular reflection, we have

$$
g^{\star}\left(t, s^{\star}, \beta\right)=\epsilon I_{b}\left(T^{\star}\left(t, s^{\star}\right)\right)+(1-\epsilon) I^{\star}\left(t, s^{\star}, \beta_{s}\right) \quad \text { for } \quad\left(t, s^{\star}, \beta\right) \in\left[0, \tau^{\star}\right] \times \partial \Omega_{-}^{\star},
$$

where $\epsilon$ is the wall emissivity (assumed to be constant), $T^{\star}\left(t, s^{\star}\right)$ is the temperature at the boundary of the medium and $\beta_{s}$ is the specular direction of radiation defined by $\beta_{s}=\beta-2\left(\beta . \mathbf{n}_{\mathbf{i}}\right) \mathbf{n}_{\mathbf{i}}$ where $\mathbf{n}_{\mathbf{i}}$ is the local outward surface normal. For an opaque wall with diffuse reflection, the boundary condition takes the following from

$g^{\star}\left(t, s^{\star}, \beta\right)=\epsilon I_{b}\left(T^{\star}\left(t, s^{\star}\right)\right)+\frac{(1-\epsilon)}{\pi} \int_{\mathbf{n}_{\mathbf{i}} \cdot \beta^{\prime}<0} I^{\star}\left(t, s, \beta^{\prime}\right)\left|\mathbf{n}_{\mathbf{i}} \cdot \beta^{\prime}\right| d \beta^{\prime} \quad\left(t, s^{\star}, \beta\right) \in\left[0, \tau^{\star}\right] \times \partial \Omega_{-}^{\star}$.

The function $g^{\star}$ is split in two parts, the emission part $g_{T}^{\star}$ and the reflected part $g_{I}^{\star}$.

$$
g^{\star}\left(t, s^{\star}, \beta\right)=g_{T}^{\star}\left(t, s^{\star}, \beta\right)+g_{I}^{\star}\left(t, s^{\star}, \beta\right) \quad\left(t, s^{\star}, \beta\right) \in\left[0, \tau^{\star}\right] \times \partial \Omega_{-}^{\star}
$$


where

$$
g_{T}^{\star}\left(t, s^{\star}, \beta\right)=\epsilon I_{b}\left(T^{\star}\left(t, s^{\star}\right)\right) \quad\left(t, s^{\star}, \beta\right) \in\left[0, \tau^{\star}\right] \times \partial \Omega_{-}^{\star}
$$

and

$$
g_{I}^{\star}\left(t, s^{\star}, \beta\right)=\frac{(1-\epsilon)}{\pi} \int_{\mathbf{n}_{\mathbf{i}} \cdot \beta^{\prime}<0} I^{\star}\left(t, s, \beta^{\prime}\right)\left|\mathbf{n}_{\mathbf{i}} \cdot \beta^{\prime}\right| d \beta^{\prime} \quad\left(t, s^{\star}, \beta\right) \in\left[0, \tau^{\star}\right] \times \partial \Omega_{-}^{\star} .
$$

It should be underlined when the wall emissivity $\epsilon=1$ we have the black walls. The radiative heat flux is defined by

$$
Q_{\text {rad }}\left(t, s^{\star}\right)=\int_{\mathcal{D}} I^{\star}\left(t, s^{\star}, \beta\right) \beta d \beta \quad \text { for }\left(t, s^{\star}\right) \in\left[0, \tau^{\star}\right] \times \Omega^{\star} .
$$

Emission and absorption of radiation by the medium lead to a radiative source term in the energy equation of the medium. It is defined by the following relations:

$$
S_{\text {rad }}^{\star}\left(t, s^{\star}\right)=\kappa\left\{G^{\star}(t, s)-4 \pi I_{b}\left(T^{\star}\left(t, s^{\star}\right)\right)\right\} \quad \text { for }\left(t, s^{\star}\right) \in\left[0, \tau^{\star}\right] \times \Omega^{\star},
$$

where $G^{\star}$ is the incident radiation intensity,

$$
G^{\star}\left(t, s^{\star}\right)=\int_{\mathcal{D}} I^{\star}\left(t, s^{\star}, \beta\right) d \beta \quad \text { for } \quad\left(t, s^{\star}\right) \in\left[0, \tau^{\star}\right] \times \Omega^{\star} .
$$

The over all energy conservation links the three different modes of heat transfer, known as conduction, radiation and convection. In this study, convection has not been considered

$$
\begin{array}{lr}
\rho c_{p} \frac{\partial T^{\star}}{\partial t}\left(t, s^{\star}\right)-k_{c} \triangle T^{\star}\left(t, s^{\star}\right)=S_{r a d}^{\star}\left(t, s^{\star}\right) & \text { for }\left(t, s^{\star}\right) \in\left[0, \tau^{\star}\right] \times \Omega^{\star} \\
T^{\star}(0, s)=T_{0}^{\star}(s) & \text { for all } s^{\star} \in \Omega^{\star} .
\end{array}
$$

The data $\rho, c_{p}$, and $k_{c}$ are the density, the specific heat capacity, and the thermal conductivity of the medium, respectively. In this work, they are assumed to be constant. The energy equation (10a)- (10b) and the RTE (2a)- (2b) are strongly coupled by the incident radiation intensity $G^{\star}$ and the temperature $T^{\star}$.

The energy equation (10a) can be written as the dimensionless form, see Appendix A:

$$
\begin{aligned}
& \frac{\partial T}{\partial \xi}-\triangle T+\theta T^{4}=\theta G \quad \text { for } \quad(\xi, s) \in[0, \tau] \times \Omega \\
& T(0, s)=T_{0}(s) \quad \text { for all } s \in \Omega
\end{aligned}
$$


where $T$ is the dimensionless temperature. We consider the Dirichlet boundary conditions, where the temperature at the boundary is known

$$
T(\xi, s)=f(\xi, s) \quad(\xi, s) \in[0, \tau] \times \partial \Omega,
$$

and the Robin thermal conditions, the flux through the boundary is defined;

$$
-\frac{\partial T}{\partial n}(\xi, s)=T(\xi, s)-f(\xi, s) \quad(\xi, s) \in[0, \tau] \times \partial \Omega .
$$

Similarly, the dimensionless form of radiative transfer equation is given by, see Appendix A:

$$
\begin{aligned}
\beta . \nabla I(\xi, s, \beta)+I(\xi, s, \beta) & =T^{4}(\xi, s), & & \text { for }(\xi, s, \beta) \in[0, \tau] \times \Omega \times \mathcal{D}, \\
I(\xi, s, \beta) & =g(\xi, s, \beta), & & \text { for } \quad(\xi, s, \beta) \in[0, \tau] \times \partial \Omega_{-} .
\end{aligned}
$$

\section{Analysis of radiative conductive heat transfer}

\subsection{Radiative Transfer Equation.}

To compute a numerical solution of the RTE, the classical $S_{N}$-discrete ordinate method is introduced [8]. The $S_{N}$ method consists of replacing the radiation intensity $I(\xi, s, \beta)$ by a discrete radiation intensity $\left(I^{1}(\xi, s), I^{2}(\xi, s)\right.$, $\left.\ldots, I^{N^{\beta}}(\xi, s)\right)$, where $I^{l}(\xi, s)=I\left(\xi, s, \beta_{l}\right)$ for all $l \in\left\{1, \ldots, N_{\beta}\right\}$. A quadrature rule was chosen $\left\{\left(\beta_{l}, w_{l}\right) l=1, \ldots, N^{\beta}\right\}$, such that:

$$
\int_{\mathcal{D}} f(\beta, s) d \beta \simeq \sum_{l=1}^{N^{\beta}} f\left(\beta_{l}, s\right) w_{l} \text { and } \sum_{l=1}^{N^{\beta}} w_{l}=4 \pi .
$$

This quadrature formula preserves a symmetry for the opposite $\beta_{l^{\prime}}=-\beta_{l}$ of discrete ordinate $\beta_{l}$ in the quadrature set, the weights are equal $w_{l^{\prime}}=w_{l}$, see [8]. The discrete radiation intensity $I^{l}$ is the solution of a $N^{\beta}$ system of partial differential equations over $\Omega$ :

$$
\left\{\begin{aligned}
\beta_{l} . \nabla I^{l}(\xi, s)+I^{l}(\xi, s) & =T^{4}(\xi, s) & & (\xi, s) \in[0, \tau] \times \Omega \\
I^{l}(\xi, s) & =g\left(\xi, s, \beta_{l}\right) & & (\xi, s) \in[0, \tau] \times \partial \Omega_{-}^{l},
\end{aligned}\right.
$$

where

$$
\partial \Omega_{-}^{l}=\left\{s \in \partial \Omega \text { such that } \beta_{l} . \mathbf{n}<0\right\} .
$$

Let $H^{r}(\Omega)$ the classical Sobolev subspace of $L^{2}(\Omega)$ functions, with norm denoted by $\|.\|_{r}$ for $r>0$, see [26]. We denote by $\digamma(\xi,)=.T^{4}(\xi,$.$) and we$ assume that $\digamma(\xi,$.$) belongs to L^{2}(\Omega)$ for all $\xi \in[0, \tau]$.We extend the boundary datum $g$ to $\partial \Omega$ by setting it to zero outside $\partial \Omega_{-}^{l}$ and we assume that

$$
g\left(\xi, ., \beta_{l}\right) \in L^{2}\left(\partial \Omega_{-}^{l}\right) \quad \text { for all }(\xi, l) \in[0, \tau] \times\left\{1, \ldots, N_{\beta}\right\} .
$$


Theorem 3.1 (Well-posedness, see [27]). The problem (16) is well-posed. Moreover, its unique solution $I^{l}(\xi,.) \in H^{1}(\Omega)$ satisfies (16) and $I^{l}(\xi,)=$. $g\left(\xi, ., \beta_{l}\right)$ a.e. in $\partial \Omega_{-}^{l}$ for all $(\xi, l) \in[0, \tau] \times\left\{1, \ldots, N_{\beta}\right\}$.

Let $\left\{I^{l}(\xi,).\right\}=\left\{I^{1}(\xi,),. I^{2}(\xi,),. \ldots, I^{N_{\beta}}(\xi,).\right\}$ and $\left\{I^{l}(\xi,).\right\} \in\left\{H^{r}(\Omega)\right\}_{l=1}^{N_{\beta}}$, where the space $\left\{H^{r}(\Omega)\right\}_{l=1}^{N_{\beta}}$ is defined such that $I^{l}(\xi,.) \in H^{r}(\Omega)$ for all $(\xi, l) \in[0, \tau] \times\left\{1, \ldots, N_{\beta}\right\}$.

Now, we proceed with the spatial discretization of the $S_{N}$ transport equation using the DG method. We consider a triangulation $\mathcal{T}_{h}$ of $\Omega$ such that

$$
\bar{\Omega}=\bigcup_{K \in \mathcal{T}_{h}} K,
$$

- Each $K$ is a triangle with nonempty internal;

- $\stackrel{\circ}{K_{1}} \cap \stackrel{\circ}{K_{2}}=\emptyset$ for each distinct $K_{1}, K_{2} \in \mathcal{T}_{h}$;

- $F=K_{1} \cap K_{2} \neq \emptyset$ then $F$ is a common face of $K_{1}$ and $K_{2}$;

- Interfaces are collected in the set $\mathcal{F}_{h}^{i n}$, and boundary faces are collected in the set $\mathcal{F}_{h}^{b}$. Henceforth, we set

$$
\mathcal{F}_{h}=\mathcal{F}_{h}^{i n} \bigcup \mathcal{F}_{h}^{b}
$$

Moreover, for any mesh element $K \in \mathcal{T}_{h}$, the set

$$
\mathcal{F}_{K}=\left\{F \in \mathcal{F}_{h} \mid F \in \partial K\right\},
$$

collects the mesh faces composing the boundary of $K$.

- $\operatorname{diam}(K)=h$ for each $K \in \mathcal{T}_{h}$ (structured mesh).

Now, an approximation space $V_{h} \subset L^{2}(\Omega)$ is introduced such that

$$
V_{h}=\left\{I_{h} \in L^{2}(\Omega) / \forall K \in \mathcal{T}_{h}, \quad I_{h_{\mid K}} \in \mathbb{P}^{k}(K)\right\},
$$

where $\mathbb{P}^{k}(K)$ denotes the set of polynomial defined in $K$ of degree less than or equal to $k$. Define:

$$
\mathbb{V}_{h}=\left\{V_{h}\right\}_{l=1}^{N_{\beta}},
$$

and denote a generic element in $\mathbb{V}_{h}$ as $I_{h}(\xi,)=.\left\{I_{h}^{l}(\xi, .)\right\}_{l=1}^{N_{\beta}}$. Due to the discontinuous nature of the spatial approximation, functions $I_{h}^{l} \in V_{h}$ are 
double-valued on interior faces. Consider an interior face $F \in \mathcal{F}_{h}^{i n}$ separating two mesh cells, $K_{1}$ and $K_{2}$. The mean value and jump of a function $I_{h}^{l}(\xi,.) \in$ $V_{h}$ are defined as follows:

$$
\left\{\left\{I_{h}^{l}(\xi, .)\right\}\right\}=\frac{1}{2}\left(I_{1}^{l}(\xi, .)+I_{2}^{l}(\xi, .)\right), \quad \llbracket I_{h}^{l}(\xi, .) \rrbracket=\left(I_{1}^{l}(\xi, .)-I_{2}^{l}(\xi, .)\right),
$$

for all $\xi \in[0, \tau]$, where $I_{1}^{l}(\xi,)=.I_{h}^{l}(\xi, .)_{\left.\right|_{K_{1}}}$ and $I_{2}^{l}(\xi,)=.I_{h}^{l}(\xi, .)_{\left.\right|_{K_{2}}}$ are the restrictions of $I_{h}^{l}(\xi,$.$) on the mesh cells K_{1}$ and $K_{2}$, respectively. The DG formulation is obtained by multiplying the $S_{N}$ equation for the direction $\beta_{l}$ with the test function $w_{h} \in \mathbb{V}_{h}$ and applying the upwind numerical fluxes $\hat{\mathcal{F}}(s)$ to approximate the quantity $\left(\beta_{l} \cdot \mathbf{n}\right) I^{l}(\xi,$.$) on the elements boundary K:$

$$
\int_{K} I_{h}^{l}(\xi, .) w_{h}-\left(\beta_{l} . \nabla_{h} w_{h}\right) I_{h}^{l}(\xi, .)+\int_{\partial K} \hat{\mathcal{F}}(s) w_{h}=\int_{\Omega} \digamma(\xi, .) w_{h}, \quad \forall \xi \in[0, \tau] .
$$

The upwind numerical flux $\hat{\mathcal{F}}(s)$ at the mesh interface $F$ from $K_{1}$ to $K_{2}$ is given by:

$$
\hat{\mathcal{F}}(s)=\beta_{l} \cdot \mathbf{n}_{\mathbf{F}}\left\{\left\{I^{l}(\xi, s)\right\}\right\}+\frac{1}{2}\left|\beta_{l} \cdot \mathbf{n}_{\mathbf{F}}\right| \llbracket I^{l}(\xi, s) \rrbracket, \quad \forall \xi \in[0, \tau],
$$

where $\mathbf{n}_{\mathbf{F}}$ is the outward unit normal to $F$ at $s$. Summing over all cells, integrating by parts a second time, and separating volume and interface terms, we obtain a global formulation. Upon introducing the bilinear form

$$
\begin{aligned}
a_{h}^{u p}\left(I_{h}(\xi, .), w_{h}\right) & =\sum_{l=1}^{N_{\beta}} w_{l}\left(\sum_{K \in \mathcal{T}_{h}}\left[\int_{K} I_{h}^{l}(\xi, s) w_{h}^{l} d s-\int_{K} I_{h}^{l}(\xi, s)\left(\beta_{l} \cdot \nabla w_{h}^{l}\right) d s\right]\right. \\
& +\int_{\partial \Omega}\left(\beta_{l} \cdot \mathbf{n}\right)^{\oplus} I_{h}^{l}(\xi, s) w_{h}^{l} d \Gamma(s)+\sum_{F \in \mathcal{F}_{h}^{i n}} \int_{F}\left(\beta_{l} \cdot \mathbf{n}_{\mathbf{F}}\right) \llbracket I_{h}^{l}(\xi, s) \rrbracket\left\{\left\{w_{h}^{l}(s)\right\}\right\} d \Gamma(s) \\
& +\sum_{F \in \mathcal{F}_{h}^{i n}} \int_{F} \frac{1}{2}\left|\beta_{l} \cdot \mathbf{n}_{\mathbf{F}}\right| \llbracket I_{h}^{l}(\xi, s) \rrbracket \llbracket w_{h}^{l} \rrbracket d \Gamma(s)-\int_{\partial \Omega}\left(\beta_{l} \cdot \mathbf{n}\right)^{\ominus} g_{I}\left(\xi, s, \beta_{l}\right) w_{h}^{l} d \Gamma(s)
\end{aligned}
$$

for all $\left(\xi, w_{h}\right) \in[0, \tau] \times \mathbb{V}_{h}$, where for a real number $x$, we define its positive and negative parts respectively as $x^{\oplus}:=\frac{1}{2}(|x|+x), x^{\ominus}:=\frac{1}{2}(|x|-x)$. By definition, the both quantities are nonnegative. Let consider the following map $l_{h}: \mathbb{V}_{h} \longrightarrow \mathbf{R}$ defined by

$$
l\left(w_{h}\right)=\sum_{l=1}^{N_{\beta}} w_{l}\left[\int_{\Omega} \digamma(\xi, s) w_{h}^{l} d s+\int_{\partial \Omega}\left(\beta_{l} \cdot \mathbf{n}\right)^{\ominus} g_{T}\left(\xi, s, \beta_{l}\right) w_{h}^{l} d \Gamma(s)\right]
$$

for all $\left(\xi, w_{h}\right) \in[0, \tau] \times \mathbb{V}_{h}$. 
The discrete ordinate DG problem is written as

$$
\text { Find } \begin{aligned}
& I_{h}(\xi, .) \in \mathbb{V}_{h} \text { such as } \\
& a_{h}^{u p}\left(I_{h}(\xi, .), w_{h}\right)=l_{h}\left(w_{h}\right) \text { for all }\left(\xi, w_{h}\right) \in[0, \tau] \times \mathbb{V}_{h} .
\end{aligned}
$$

At the discrete level, we consider an approximation space given by the broken polynomial space $V_{h}=\mathbb{P}^{k}(K)$, for all $K \in \mathcal{T}_{h}$. The global enumeration of the degrees of freedom is such that the local degrees of freedom are numbered contiguously for each mesh element. This leads to a basis for $V_{h}$ of the form

$$
\left\{\left\{\phi_{i}^{K}\right\}_{i \in D_{K}}\right\}_{K \in \mathcal{T}_{h}},
$$

where the set $D_{K}=\left\{1, \ldots, N_{d}^{K}\right\}$ collects the local indices of the $N_{d}^{K}$ degrees of freedom for the mesh element $K$ ( we assumed that all element has the same degree $N_{d}^{K}$ ).

The global matrix can be block-portioned in the form

$$
\mathbb{A}_{h}^{D G}=\left[\begin{array}{cccc}
A_{h}^{K_{1} K_{1}} & A_{h}^{K_{1} K_{2}} & \cdots & A_{h}^{K_{1} K_{N}} \\
A_{h}^{K_{1} K_{1}} & A_{h}^{K_{2} K_{2}} & \cdots & A_{h}^{K_{1} K_{2}} \\
\vdots & \ddots & \ddots & \vdots \\
A_{h}^{K_{N} K_{1}} & A_{h}^{K_{N} K_{2}} & \cdots & A_{h}^{K_{N} K_{N}}
\end{array}\right]
$$

where for all $K_{l}, K_{m} \in \mathcal{T}_{h}$,

$$
A_{h}^{K_{m} K_{l}}=\left[a_{h}^{u p}\left(\phi_{i}^{K_{m}}, \phi_{j}^{K_{l}}\right)\right] \in \mathbf{R}^{N_{d}^{K}} \times \mathbf{R}^{N_{d}^{K}} .
$$

We split the discrete bilinear form $a_{h}^{u p}$ into volume, interface, and boundary face contributions as follows: For all $I_{h}(\xi,),. w_{h} \in \mathbb{V}_{h}$,

$$
a_{h}^{u p}\left(I_{h}(\xi, .), w_{h}\right)=a_{h}^{v}\left(I_{h}(\xi, .), w_{h}\right)+a_{h}^{i f}\left(I_{h}(\xi, .), w_{h}\right)+a_{h}^{b}\left(I_{h}(\xi, .), w_{h}\right)
$$

where

$$
\begin{aligned}
a_{h}^{v}\left(I_{h}(\xi, .), w_{h}\right)=\sum_{l=1}^{N_{\beta}} w_{l}( & \sum_{K \in \mathcal{T}_{h}}\left[\int_{K} I_{h}^{l}(\xi, s) w_{h}^{l} d s-\int_{K} I_{h}^{l}(\xi, s)\left(\beta_{l} . \nabla w_{h}^{l}\right) d s\right], \\
a_{h}^{i f}\left(I_{h}(\xi, .), w_{h}\right)= & \sum_{F \in \mathcal{F}_{h}^{i n}} \int_{F}\left(\beta_{l} \cdot \mathbf{n}_{\mathbf{F}}\right) \llbracket I_{h}^{l}(\xi, s) \rrbracket\left\{\left\{w_{h}^{l}(s)\right\}\right\} d \Gamma(s) \\
& +\sum_{F \in \mathcal{F}_{h}^{i n}} \int_{F} \frac{1}{2}\left|\beta_{l} \cdot \mathbf{n}_{\mathbf{F}}\right| \llbracket I_{h}^{l}(\xi, s) \rrbracket \llbracket w_{h}^{l} \rrbracket d \Gamma(s)
\end{aligned}
$$


and

$$
a_{h}^{b}\left(I_{h}(\xi, .), w_{h}\right)=\int_{\partial \Omega}\left(\beta_{l} \cdot \mathbf{n}\right)^{\oplus} I_{h}^{l}(\xi, s) w_{h}^{l} d \Gamma(s)-\int_{\partial \Omega}\left(\beta_{l} \cdot \mathbf{n}\right)^{\ominus} g_{I}\left(\xi, s, \beta_{l}\right) w_{h}^{l} d \Gamma(s) .
$$

Each summation yields a loop over the corresponding mesh entities to assemble local contributions into the global matrix. The local matrix stemming from the volume contribution of a generic mesh element $K \in \mathcal{T}_{h}$ is

$$
A^{K}=\left[a_{h}^{v}\left(\phi_{i}^{K}, \phi_{j}^{K}\right)\right] \in \mathbf{R}^{N_{d}^{K}} \times \mathbf{R}^{N_{d}^{K}}
$$

and it contributes to the diagonal block $A^{K K}$ of the global matrix $A$. An interface $F \in \mathcal{F}_{h}^{\text {in }}$ contributes to four blocks of the matrix $A$, and the local matrix stemming from the interface contribution can be block-partitioned in the form

$$
\mathbb{A}^{F}=\left[\begin{array}{ll}
A_{h}^{K_{1} K_{1}} & A_{h}^{K_{1} K_{2}} \\
A_{h}^{K_{1} K_{1}} & A_{h}^{K_{2} K_{2}}
\end{array}\right]
$$

where $F=\partial K_{1} \bigcap \partial K_{2}$,

$$
A_{h}^{K_{m} K_{n}}=\left[a_{h}^{i f}\left(\phi_{i}^{K_{n^{\prime}}}, \phi_{j}^{K_{m^{\prime}}}\right)\right] \in \mathbf{R}^{N_{d}^{K}} \times \mathbf{R}^{N_{d}^{K}}
$$

$\forall m, n \in\{1,2\}$ and $n^{\prime}, m^{\prime} \in\{1, \ldots, N\}$ are the indices of $K_{1}, K_{2}$ in the global enumeration of mesh elements. Finally, a boundary face $F \in \mathcal{F}_{h}^{b}$ contributes through the local matrix

$$
A^{F}=\left[a_{h}^{b}\left(\phi_{i}^{K}, \phi_{j}^{K}\right)\right] \in \mathbf{R}^{N_{d}^{K}} \times \mathbf{R}^{N_{d}^{K}}
$$

where $F=\partial K_{1} \bigcap \partial K_{2}$.

We can write (24) in the matrix form as

$$
\mathbb{A}_{h}^{D G} \mathbb{I}_{h}^{l}=\mathbb{F}_{h}^{D G}
$$

where the unknown $\mathbb{I}_{h}^{l}$ of the system (33) is defined by

$$
\mathbb{I}_{h}^{l}=\left(I_{h, 1}^{1}, . ., I_{h, N_{d}^{K}}^{1}, \ldots, I_{h, 1}^{N_{t}}, . ., I_{h, N_{d}^{K}}^{N_{t}}\right)^{T}
$$

for $l \in\left\{1, \ldots, N_{\beta}\right\}$. The second member $\mathbb{F}_{h}^{D G}$ is given by the first part of the map (23) and the emission part $g_{T}$ where the reflected part $g_{I}$ is taken into account in the matrix $\mathbb{A}_{h}^{D G}$. A direct method for sparse matrices was implemented to solve linear system (33). Finally, we note that the matrix 
assembly implementation and the basis functions selection in broken polynomial spaces are discussed in [27, p. 343, Appendix A: Implementation] and [13].

Now, to prove that problem (24) without the reflected boundary part has a unique solution, we consider in $\mathbb{V}_{h}$ the following norm:

$$
\|I\|_{h}^{2}=\sum_{l=1}^{N_{\beta}} w_{l}\left[\left\|I^{l}\right\|_{L^{2}(\Omega)}^{2}+\int_{\partial \Omega} \frac{1}{2}\left|\beta_{l} \cdot \mathbf{n}\right| I^{l^{2}} d \Gamma(s)+\sum_{F \in \mathcal{F}_{h}^{i n}} \int_{F} \frac{1}{2}\left|\beta_{l} \cdot \mathbf{n}_{\mathbf{F}}\right| \llbracket I^{l} \rrbracket^{2} d \Gamma(s)\right],
$$

for all $I \in \mathbb{V}_{h}$. Then, we deduce the following stability result.

Lemma 3.2. Let $\xi \in[0, \tau]$, then

$$
a_{h}^{u p}\left(I_{h}(\xi, .), I_{h}(\xi, .)\right)=\left\|I_{h}(\xi, .)\right\|_{h}^{2} \quad \text { for all } I_{h}(\xi, .) \in \mathbb{V}_{h} .
$$

Proof. Let $\xi \in[0, \tau]$, by definition of $a_{h}^{u p}(.,$.$) we have for any I_{h}(\xi,.) \in \mathbb{V}_{h}$,

$$
\begin{aligned}
a_{h}^{u p}\left(\left\{I_{h}\right\}(\xi),\left\{I_{h}\right\}(\xi)\right) & =\sum_{l=1}^{N_{\beta}} w_{l} \sum_{K \in \mathcal{T}_{h}}\left[\int_{K}\left(I_{h}^{l}(\xi, s)\right)^{2} d s-\int_{K} I_{h}^{l}(\xi, s)\left(\beta_{l} . \nabla I_{h}^{l}(\xi, s)\right) d s\right] \\
& +\int_{\partial \Omega}\left(\beta_{l} \cdot \mathbf{n}\right)^{\oplus}\left(I_{h}^{l}(\xi, s)\right) 2 d \Gamma(s)+\sum_{F \in \mathcal{F}_{h}^{\text {in }}} \int_{F}\left(\beta_{l} \cdot \mathbf{n}_{\mathbf{F}}\right) \llbracket I_{h}^{l}(\xi, s) \rrbracket\left\{\left\{I_{h}^{l}(\xi, s)\right\}\right\} d \Gamma(s) \\
& +\sum_{F \in \mathcal{F}_{h}^{\text {in }}} \int_{F} \frac{1}{2}\left|\beta_{l} \cdot \mathbf{n}_{\mathbf{F}}\right| \llbracket I_{h}^{l}(\xi, s) \rrbracket \llbracket I_{h}^{l}(\xi, s) \rrbracket d \Gamma(s) \forall\left(\xi, w_{h}\right) \in[0, \tau] \times \mathbb{V}_{h},
\end{aligned}
$$

Using the integration by parts formula, it follows

$$
\begin{aligned}
a_{h}^{u p}\left(\left\{I_{h}\right\}(\xi),\left\{I_{h}\right\}(\xi)\right) & =\sum_{l=1}^{N_{\beta}} w_{l} \sum_{K \in \mathcal{T}_{h}}\left[\int_{K}\left(I_{h}^{l}(\xi, s)\right)^{2} d s-\frac{1}{2} \int_{\partial K}\left(\beta_{l} \cdot \mathbf{n}_{\mathbf{K}}\right)\left(I_{h}^{l}(\xi, s)\right)^{2} d s\right] \\
& +\int_{\partial \Omega}\left(\beta_{l} \cdot \mathbf{n}\right)^{\oplus}\left(I_{h}^{l}(\xi, s)\right)^{2} d \Gamma(s)+\sum_{F \in \mathcal{F}_{h}^{i n}} \int_{F}\left(\beta_{l} \cdot \mathbf{n}_{\mathbf{F}}\right) \llbracket I_{h}^{l}(\xi, s) \rrbracket\left\{\left\{I_{h}^{l}(\xi, s)\right\}\right\} d \Gamma(s) \\
& +\sum_{F \in \mathcal{F}_{h}^{i n}} \int_{F} \frac{1}{2}\left|\beta_{l} \cdot \mathbf{n}_{\mathbf{F}}\right| \llbracket I_{h}^{l}(\xi, s) \rrbracket \llbracket I_{h}^{l}(\xi, s) \rrbracket d \Gamma(s) \forall\left(\xi, w_{h}\right) \in[0, \tau] \times \mathbb{V}_{h},
\end{aligned}
$$

The second term on the right hand side can be reformulated. Indeed, the continuity of $\beta_{l}$ across the interfaces leads to

$$
\sum_{K \in \mathcal{K}_{h}} \int_{\partial K} \frac{1}{2}\left(\beta_{l} \cdot \mathbf{n}_{K}\right)\left(I_{h}^{l^{2}}(\xi, s)\right)=\sum_{F \in \mathcal{F}_{h}^{i n}} \int_{F} \frac{1}{2}\left(\beta_{l} \cdot \mathbf{n}_{F}\right) \llbracket I_{h}^{l}(\xi, s)^{2} \rrbracket+\sum_{F \in \mathcal{F}_{h}^{b}} \int_{F} \frac{1}{2}\left(\beta \cdot \mathbf{n}_{F}\right) I_{h}^{l^{2}}(\xi, s) .
$$


For all $F \in \mathcal{F}_{h}^{\text {in }}$, where $\mathbf{n}_{\mathbf{K}_{\mathbf{1}}}$ is the unit normal to $F$ at $s$ pointing from $K_{1}$ to $K_{2} . I_{i}^{l}(\xi, s)=I_{h}^{l}(\xi, s)_{\left.\right|_{K_{i}}}, i \in\{1,2\}$ it holds

$$
\begin{aligned}
\frac{1}{2} \llbracket I_{h}^{l}(\xi, .) \rrbracket=\frac{1}{2}\left(I_{1}^{l^{2}}(\xi, .)-I_{2}^{l_{2}}(\xi, .)\right)= & \frac{1}{2}\left(I_{1}^{l}(\xi, .)-I_{2}^{l}(\xi, .)\right)\left(I_{1}^{l}(\xi, .)+I_{2}^{l}(\xi, .)\right) \\
& =\llbracket I_{h}^{l}(\xi, .) \rrbracket\left\{\left\{I_{h}^{l}(\xi, .)\right\}\right\} .
\end{aligned}
$$

We obtain

$$
\begin{aligned}
a_{h}^{u p}\left(I_{h}(\xi, .), I_{h}(\xi, .)\right) & =\sum_{l=1}^{N_{\beta}} w_{l}\left\|I_{h}^{l}(\xi, .)\right\|_{L^{2}(\Omega)}^{2}+\frac{1}{2} \int_{\partial \Omega}\left|\beta_{l} \cdot \mathbf{n}\right|\left(I_{h}^{l}(\xi, s)\right)^{2} d \Gamma(s) \\
& +\sum_{F \in \mathcal{F}_{h}^{\text {in }}} \int_{F} \frac{1}{2}\left|\beta_{l} \cdot \mathbf{n}_{\mathbf{F}}\right| \llbracket I_{h}^{l}(\xi, s) \rrbracket^{2} d \Gamma(s) .
\end{aligned}
$$

Then,

$$
a_{h}^{u p}\left(I_{h}(\xi, .), I_{h}(\xi, .)\right)=\left\|I_{h}(\xi, .)\right\|_{h} \quad \text { for all } \xi \in[0, \tau] .
$$

Theorem 3.3. The problem (24) has a unique solution in $\mathbb{V}_{h}$.

Proof. $l_{h}$ denotes the continuous map from $\mathbb{V}_{h}$ to $\mathbf{R}$. Using the lemma 3.2 we deduce that (24) has a unique solution.

To give an error estimate for the discrete ordinate and the DG method considered in this work we applied the methodology used in [28].

Let $\left\{I^{l}(\xi,).\right\} \in\left\{H^{r}(\Omega\}_{l=1}^{N_{\beta}}\right.$ for $r>0$. If $\left\{I^{l}(\xi,).\right\}$ satisfies:

$$
a_{h}^{u p}\left(\left\{I^{l}(\xi, .)\right\}, w_{h}\right)=l_{h}\left(w_{h}\right) \text { for all }\left(\xi, w_{h}\right) \in[0, \tau] \times \mathbb{V}_{h} .
$$

The discrete-ordinate DG method has the following error estimate

$$
\left\|\left\{I^{l}(\xi, .)\right\}-\left\{I_{h}(\xi, .)\right\}\right\|_{h} \leqslant C h^{\min \{r, k+1\}-\frac{1}{2}}\left[\sum_{l=1}^{N_{\beta}} w_{l}\left\|\left\{I^{l}(\xi, .)\right\}\right\|_{r}^{2}\right]^{\frac{1}{2}} \quad \text { for all } \xi \in[0, \tau] .
$$

with $C$ a positive constant and $k$ is the degree of the polynomial of approximation. Now, we can give a $L^{2}(\Omega)$ error estimate. To this end, we introduce the norm:

$$
\left\|\left|\{I(\xi, .)\}-\left\{I_{h}(\xi, .)\right\} \|\right|=\left[\sum_{l=1}^{N_{\beta}} w_{l}\left\|\left\{I^{l}(\xi, .)\right\}-\left\{I_{h}^{l}(\xi, .)\right\}\right\|_{L^{2}(\Omega)}^{2}\right]^{\frac{1}{2}} \quad \text { for all } \xi \in[0, \tau] .\right.
$$

Thanks to the estimate (41) we deduce the following result 
Lemma 3.4. Assume that $\left\{I^{l}(\xi,).\right\} \in\left\{H^{r}(\Omega\}^{N_{\beta}}\right.$ for $r>0$ is the solution of (40), $I_{h} \in \mathbb{V}_{h}$ is solution of (24) and that the numerical quadrature satisfies (15). Then, the discrete-ordinate $D G$ method, leads to

$$
\left\|\mid\{I(\xi, .)\}-\left\{I_{h}(\xi, .)\right\}\right\| \leqslant C_{1} h^{\min \{r, k+1\}-\frac{1}{2}}\left[\sum_{l=1}^{N_{\beta}} w_{l}\left\|\left\{I^{l}(\xi, .)\right\}\right\|_{r}^{2}\right]^{\frac{1}{2}} \quad \text { for all } \xi \in[0, \tau],
$$

where $C_{1}$ is a positive constant.

\subsection{Energy equation}

Let $(., .)_{\Omega}$ and $(., .)_{\partial \Omega}$ be the inner product in $L^{2}(\Omega)$ and $L^{2}(\partial \Omega)$ respectively. To consider a weak solution of the equation (11a), the following space is introduced :

$$
\mathcal{V}=\left\{T \in L^{2}\left(0, \tau, H^{1}(\Omega)\right) \text { such that } T(\xi, s)=f(\xi, s) \text { for }(\xi, s) \in[0, \tau] \times \partial \Omega\right\} .
$$

The weak formulation of equation (11a) for Dirichlet boundary conditions (12) reads: Find $T \in \mathcal{V}$ such that

$$
\left(\frac{d T}{d \xi}, \varphi\right)_{\Omega}+(\nabla T, \nabla \varphi)_{\Omega}+\theta\left(T^{4}, \varphi\right)_{\Omega}=\theta(G, \varphi)_{\Omega},
$$

for all $\varphi \in H_{0}^{1}(\Omega)$ and satisfying the initial condition:

$$
(T(0, s), \varphi)_{\Omega}=\left(T_{0}, \varphi\right)_{\Omega} \quad \forall \varphi \in L^{2}(\Omega) .
$$

In the case of Robin boundary conditions (13), we have:

Find $T \in L^{2}\left(0, \tau, H^{1}(\Omega)\right)$ such that

$$
\left(\frac{d T}{d \xi}, \varphi\right)_{\Omega}+(\nabla T, \nabla \varphi)_{\Omega}+\theta\left(T^{4}, \varphi\right)_{\Omega}+(T, \varphi)_{\partial \Omega}=\theta(G, \varphi)_{\Omega}+(f, \varphi)_{\partial \Omega},
$$

for all $\varphi \in H^{1}(\Omega)$ and satisfying the initial condition:

$$
(T(0, s), \varphi)_{\Omega}=\left(T_{0}, \varphi\right)_{\Omega} \quad \forall \varphi \in L^{2}(\Omega) .
$$

A proof of the existence and uniqueness of the solution of system (45)-(47) is given in [23].

\subsection{Numerical approach of the energy equation}

Let

$$
W_{h}=\left\{v_{h} \in \mathcal{C}^{0}(\Omega) / \forall K \in \mathcal{T}_{h}, \quad v_{h_{\left.\right|_{K}}} \in \mathbb{P}^{k}(K)\right\},
$$


be a family of finite element subspaces of $H^{1}(\Omega)$. The finite dimensional space $W_{N^{b s}}=\operatorname{vect}\left\{\phi_{1}, \phi_{2}, \ldots, \phi_{N^{b s}}\right\}$. We denote by $M_{i}$ a node of the mesh $\mathcal{T}_{h}$. In the Dirichlet boundary conditions case, we denote by $\mathcal{E}$ the set of points on the edge $\left(\mathcal{E}=\left\{i\right.\right.$ such that $\left.\left.M_{i} \in \partial \Omega\right\}\right), N^{b e}=\operatorname{Card}(\mathcal{E})$ and $\mathcal{I}=\left\{1, \ldots, N^{b s}\right\} \backslash$ $\mathcal{E}$. To construct a finite element approximation of the temperature $T$, we introduce:

$$
T_{h}(\xi, s)=\sum_{i=1}^{N^{b s}} T_{h i}(\xi) \phi_{i}(s) .
$$

Then, a semi-discrete finite element approximation of the weak formulation (45) is: Find $T_{h}:[0, \tau] \rightarrow T_{h}(\xi,.) \in W_{N^{b s}}$ such that $T_{h}\left(\xi, M_{j}\right)=f\left(\xi, M_{j}\right)$ for all $j \in \mathcal{E}$

$$
\left(\frac{d T_{h}}{d \xi}, \phi_{i}\right)_{\Omega}+\left(\nabla T_{h}, \nabla \phi_{i}\right)_{\Omega}+\theta\left(T_{h}^{4}, \phi_{i}\right)_{\Omega}=\theta\left(G_{h}, \phi_{i}\right)_{\Omega} \quad \forall \phi_{i} \in W_{N^{b s}}
$$

for all $i \in \mathcal{I}$ and satisfying the initial condition:

$$
\left(T_{h}, \phi_{i}\right)_{\Omega}=\left(T_{0}, \phi_{i}\right)_{\Omega} \quad \forall \phi_{i} \in W_{N}^{b s}
$$

In the Robin boundary conditions case, a semi-discrete finite element approximation of the weak formulation (47) is: Find $T_{h}:[0, \tau] \rightarrow T_{h}(\xi,.) \in W_{N^{b s}}$ such that

$$
\begin{aligned}
\left(\frac{d T}{d \xi}, \phi_{i}\right)_{\Omega}+\left(\nabla T_{h}, \nabla \phi_{i}\right)_{\Omega}+ & \theta\left(T_{h}{ }^{4}, \phi_{i}\right)_{\Omega}+\left(T_{h}, \phi_{i}\right)_{\partial \Omega} \\
& =\theta\left(G, \phi_{i}\right)_{\Omega}+\left(f, \phi_{i}\right)_{\partial \Omega} \quad \forall \phi_{i} \in W_{N^{b s}},
\end{aligned}
$$

and satisfying the initial condition:

$$
\left(T_{h}, \phi_{i}\right)_{\Omega}=\left(T_{0}, \phi_{i}\right)_{\Omega} \quad \forall \phi_{i} \in W_{N^{b s}} .
$$

We used the Crank-Nicolson scheme for time discretization, Let $\triangle \xi=\frac{\tau}{N}$, the equation (51) can be written in the following form

$$
\begin{aligned}
&\left(\frac{T_{h}^{n+1}-T_{h}^{n}}{\triangle \xi}, \phi_{i}\right)_{\Omega}+\left(\nabla\left(\frac{\left.T_{h}^{n+1}+T_{h}^{n}\right)}{2}, \nabla \phi_{i}\right)_{\Omega}+\theta\left(\frac{T_{h}^{n+1^{4}}+T_{h}^{n 4}}{2}, \phi_{i}\right)_{\Omega}\right. \\
&=\theta\left(G^{n}, \phi_{i}\right)_{\Omega} \quad \forall \phi_{i} \in W_{N^{b s}} .
\end{aligned}
$$

The Newton method is employed for analyzing the nonlinearity,

$$
T_{h}^{{ }^{n+1}}{ }^{4} \simeq T_{h}^{n 4}+4 T_{h}^{n 3}\left(T_{h}^{n+1}-T_{h}^{n}\right),
$$


then, the equation (55) can be written as

$$
\begin{aligned}
\left(\frac{T_{h}^{n+1}-T_{h}^{n}}{\triangle \xi}, \phi_{i}\right)_{\Omega}+ & \left(\nabla\left(\frac{\left.T_{h}^{n+1}+T_{h}^{n}\right)}{2}\right), \nabla \phi_{i}\right)_{\Omega}+\theta\left(\left(-T_{h}^{n 4}+2 T_{h}^{n 3} T_{h}^{n+1}\right), \phi_{i}\right)_{\Omega} \\
= & \theta\left(G^{n}, \phi_{i}\right)_{\Omega} \quad \forall \phi_{i} \in W_{N^{b s}} .
\end{aligned}
$$

In the same way, the equation (53) can be written as

$$
\begin{aligned}
& \left(\frac{T_{h}^{n+1}-T_{h}^{n}}{\triangle \xi}, \phi_{i}\right)_{\Omega}+\left(\nabla\left(\frac{\left.T_{h}^{n+1}+T_{h}^{n}\right)}{2}\right), \nabla \phi_{i}\right)_{\Omega}+\theta\left(\left(-T_{h}^{n 4}+2 T_{h}^{n^{3}} T_{h}^{n+1}\right), \phi_{i}\right)_{\Omega} \\
& +\left(\frac{\left(T_{h}^{n+1}+T_{h}^{n}\right)}{2}, \phi_{i}\right)_{\partial \Omega}=\theta\left(G^{n}, \phi_{i}\right)_{\Omega}+\left(f^{n}, \phi_{i}\right)_{\partial \Omega} \quad \forall \phi_{i} \in W_{N^{b s}}
\end{aligned}
$$

where $T_{h}^{n}$ is the approximate value of $T_{h}$ at time $\xi^{n}=n . \triangle \xi$, for all $n \in$ $\{0,1, \ldots, N\}$.

Let us denote by $\tilde{T}_{h}^{n}=\left[T_{h i}^{n}\right]_{i=1}^{N^{b s}}, \tilde{G}^{n}=\left[G_{h i}^{n}\right]_{i=1}^{N^{b s}}$ and $\tilde{F}^{n}=\left[f_{h i}^{n}\right]_{i=1}^{N^{b s}}$ where $G_{h i}^{n}$ and $f_{h i}^{n}$ are given by

$$
G_{h i}^{n}=\left(G^{n}, \phi_{i}\right)_{\partial \Omega} \quad f_{h i}^{n}=\left(f^{n}, \phi_{i}\right)_{\partial \Omega} .
$$

Then, we obtain the following system

$$
\begin{aligned}
& \mathcal{M}_{h}\left(\frac{\tilde{T}_{h}^{n+1}-\tilde{T}_{h}^{n}}{\triangle \xi}\right)+\mathcal{A}_{h}\left(\frac{\tilde{T}_{h}^{n+1}+\tilde{T}_{h}^{n}}{2}\right)+\theta \mathcal{M}_{h}\left(-\tilde{T}_{h}^{n^{4}}+2 \tilde{T}_{h}^{n^{3}} \tilde{T}_{h}^{n+1}\right) \\
& +\mathcal{M}_{h}\left(\frac{\tilde{T}_{h}^{n+1}+\tilde{T}_{h}^{n}}{2}\right)=\theta \tilde{G}^{n}+\tilde{F}^{n}
\end{aligned}
$$

for all $n \in\{0,1, \ldots, N\}$, where $\mathcal{M}_{h}$ and $\mathcal{A}_{h}$ denote respectively the mass and stiffness matrix of finite elements method. A proof of the existence and uniqueness of the solution of discrete system (58) is given in [24]. A priori estimates of numerical error are given in [25].

Given an initial condition $T_{0}$, at each time step: first we solve the RTE, second we compute the the incident radiation intensity $G$ and solve the energy equation. The described iteration are performed until a time $\tau_{1}$ such that the difference in $L^{2}$ norm between the solutions of the energy equation at time $\tau_{1}$ and time $\tau_{1}+\triangle \xi$ is less than $10^{-5}$.

\section{Results and discussion}

\subsection{Radiative heat transfer in a square enclosure.}

To show the performance of the DG method considered in this work we will first present some test cases. The first case consists of calculating the $L^{2}$ 
norm of the error for a particular form of the RTE solved for a prescribed direction $\beta$ in a square enclosure. This equation has the following form:

$$
\left\{\begin{aligned}
\beta . \nabla I+I=\digamma & \text { in } \Omega . \\
I=0 & \text { on } \partial \Omega_{-},
\end{aligned}\right.
$$

where

$$
\partial \Omega_{-}=\{s \in \partial \Omega \text { such that } \beta . \mathbf{n}<0\},
$$

and

$$
\left\{\begin{aligned}
\beta & =\left(\cos \left(\frac{\pi}{4}\right), \sin \left(\frac{\pi}{4}\right)\right) \\
\digamma & =\cos \left(\frac{\pi}{4}\right) \frac{\pi}{2} \sin \left(\pi s_{2}\right) \sin \left(\frac{\pi}{2}\left(1-s_{1}\right)\right)+\sin \left(\frac{\pi}{4}\right) \pi \cos \left(\pi s_{2}\right) \cos \left(\frac{\pi}{2}\left(1-s_{1}\right)\right) \\
& +\sin \left(\pi s_{2}\right) \cos \left(\frac{\pi}{2}\left(1-s_{1}\right)\right),
\end{aligned}\right.
$$

which has a smooth exact solution given by $\sin \left(\pi s_{2}\right) \cos \left(\frac{\pi}{2}\left(1-s_{1}\right)\right) \in \mathcal{C}^{\infty}(\Omega)$, see Figure 1.

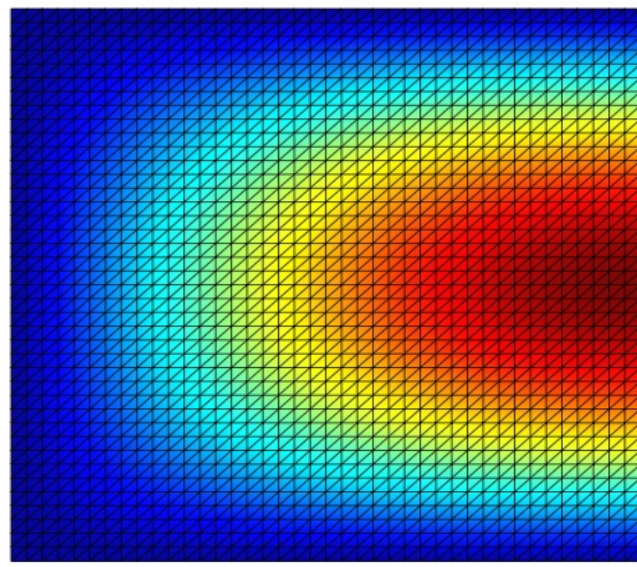

0.5

Figure 1: The exact solution in mesh of unit square enclosure.

Figure 2 shows the $L^{2}$ norm of the error for different orders of approximation of the DG method. Our results were found to comply with the theoretical result (41) for the smooth solution $I$. When we took $r$ sufficiently higher, the 


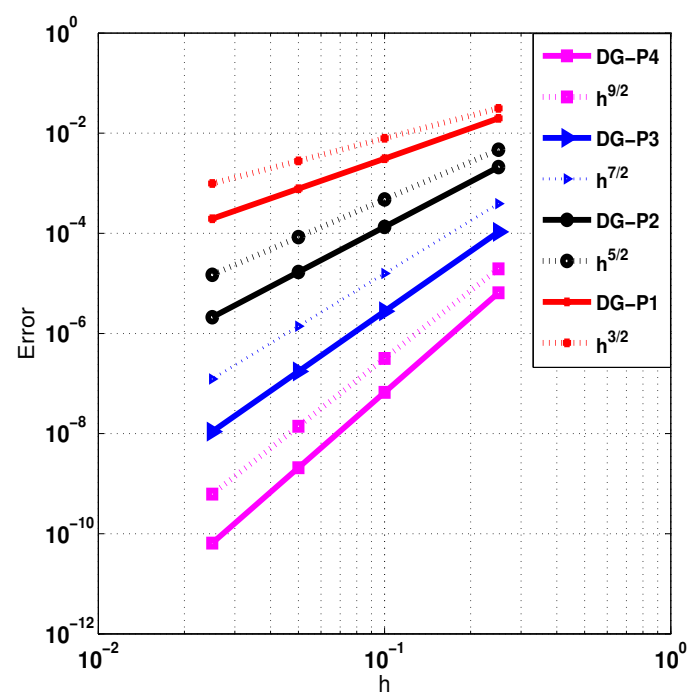

Figure 2: $L^{2}$ norm of the error for the upwind DG method.

curve of the error with the polynomial approximation $P_{k}$ for $k \in\{1, \ldots, 4\}$ was found to be proportional to $h^{k+\frac{1}{2}}\left(k+\frac{1}{2}=\min \left(k+\frac{1}{2}, r\right)\right.$ for a sufficiently higher value of $r$ ). In the second test case, we assumed that the medium to be grey in a square enclosure. The enclosure was assumed to be filled with an absorbing medium at constant temperature $T_{m}=100 \mathrm{~K}$ and the walls assumed to be black and cold $T_{N}=T_{E}=T_{S}=T_{W}=0 K$ where $T_{m}$ was the temperature of the medium and $T_{N}, T_{E}, T_{S}, T_{W}$ are the temperatures at the boundary of the medium (North, East, South and West respectively). Numerical simulations were performed using the $S_{2}, S_{4}$ and $S_{6}$ quadratures and with a structured triangular grid composed of 441 nodes. Figure 3 shows the dimensionless radiative heat flux $\frac{Q_{\text {rad }}}{\sigma_{B} T_{r e f}^{4}}$ for three values of the absorption coefficient $\left(\kappa=10, \kappa=1\right.$ and $\left.\kappa=0.1 \mathrm{~m}^{-1}\right)$. The numerical solution was found to be very close to the exact solution in passing $S_{2}$ to $S_{4}$ and from $S_{4}$ to $S_{6}$ respectively . Our results with $S_{6}$ comply well with the exact solution given in [29]. When the medium was more absorbing $\left(\kappa=10 \mathrm{~m}^{-1}\right)$, the heat flux was found to be greater and tended towards the heat flux of the blackbody at temperature of the medium. When $\kappa$ decreased, the heat flux tended towards zero since the walls were cold. Our results show that the $S_{6}$ quadrature already gives a better approximation than the results presented 
in $[30,31]$. In [31], the author shows that the error estimate was proportional to the inverse of the number of discrete directions $N_{\beta}$. In figure 4 the numerical results were obtained using the $S_{4}$ angular discretization and for two values of the absorption coefficient $\kappa=2 \mathrm{~m}^{-1}$ and $\kappa=10 \mathrm{~m}^{-1}$. Figure 4 shows the numerically computed dimensionless incident radiative heat flux. Accuracy was found to increase when the polynomial approximation degree was higher which again complies with the theoretical results.

\subsection{Combined radiative and conductive heat transfer in a square enclosure.}

In this section, we shall present the numerical results for the coupled radiative-conductive heat transfer problem. In this case, the $S_{4}$ angular discretization was used to reduce the computational time while maintaining a reasonable level of accuracy. The problems presented were solved with a constant dimensionless time step set to $\triangle \xi=5 \cdot 10^{-4}$. The reference temperature $T_{\text {ref }}$ was assumed to be the hot temperature $T_{\text {ref }}=1000 \mathrm{~K}$. In addition, all calculations were performed with the absorption coefficient $\kappa=1 \mathrm{~m}^{-1}$ and in all cases, iterations were terminated when the steady state conditions were achieved with a tolerance set to $10^{-5}$. For each test case, CPU time is indicated. Initially, the medium was assumed to be at a constant temperature equal to $T_{0}^{\star}=500 \mathrm{~K}$. The south surface (hot surface) and the other faces were kept constant over time. The thermal boundary conditions considered in the first test case were non-homogeneous Dirichlet conditions (12) and our aim in using these was to check our results against those in literature on subject. The Robin boundary conditions (13), were also considered. All calculations were performed on a Mac OS X version 10.6.6 2.22GHz Intel Core 2 Duo using the MATLAB software.

\subsubsection{Non-homogeneous thermal Dirichlet boundary conditions.}

In this section, we used the finite element method with an order of approximation equal to 2 (EFP2) to solve the energy equation and the DG method with an order of approximation equal to 2 (DGP2) was used to solve the RTE. The simulations were performed for two types of mesh, a structured triangular grid composed of 1600 nodes (mesh1, $\mathrm{h}=0.025$ ), see figure 5-(a) and a refined mesh in high-temperature region composed of 1534 nodes (mesh2), figure 5-(b). Under these conditions, the computational times required by the program to converge to the steady state are approximately $2 \mathrm{~h}$ when $N_{s}=1$ and $N_{s}=0.1$. 


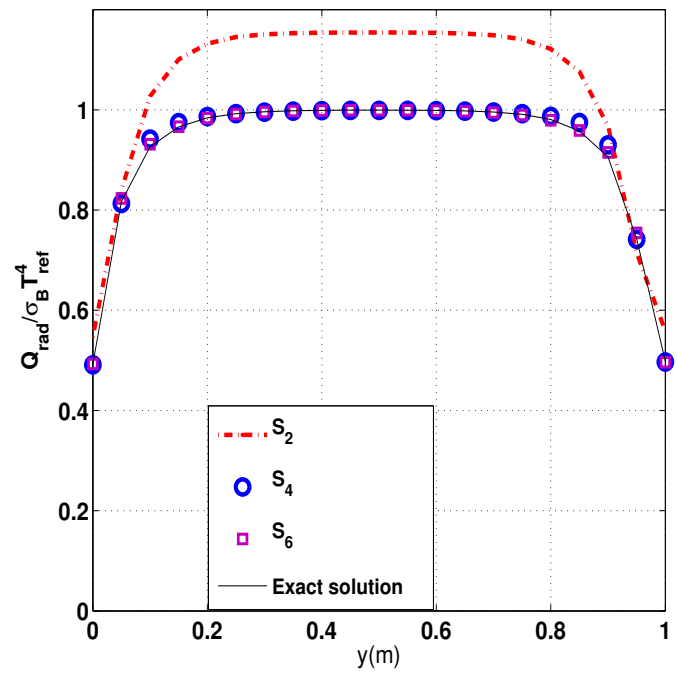

(a) $\kappa=10 m^{-1}$

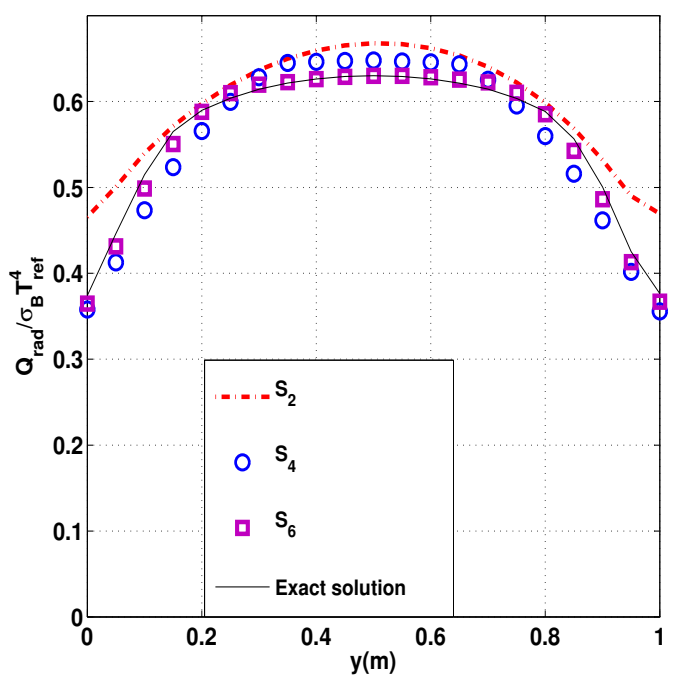

(b) $\kappa=1 m^{-1}$

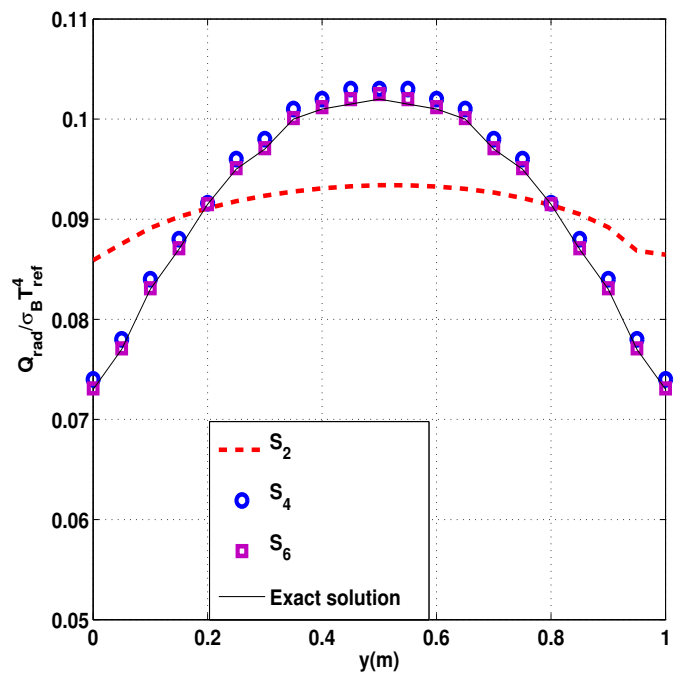

(c) $\kappa=0.1 m^{-1}$

Figure 3: Dimensionless radiative heat flux along the centerline of the south boundary, $D G-P^{1}$ 


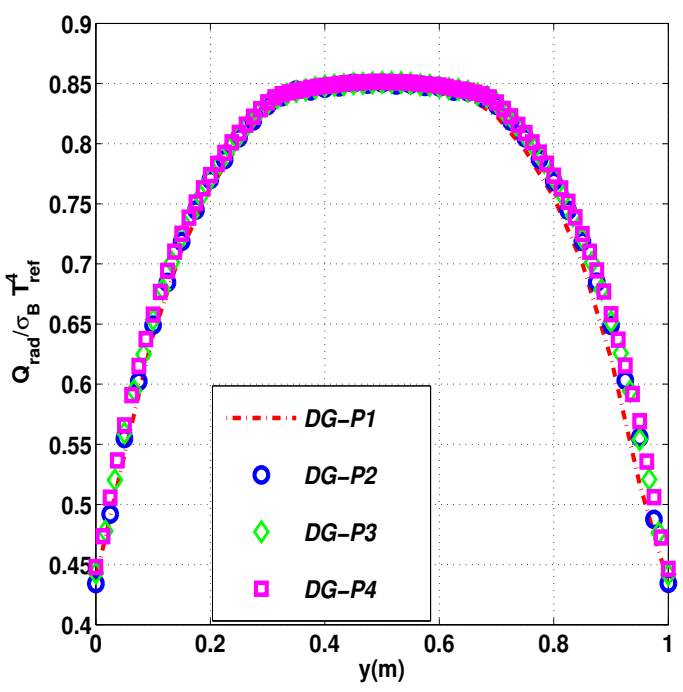

(a) $\kappa=2 m^{-1}$

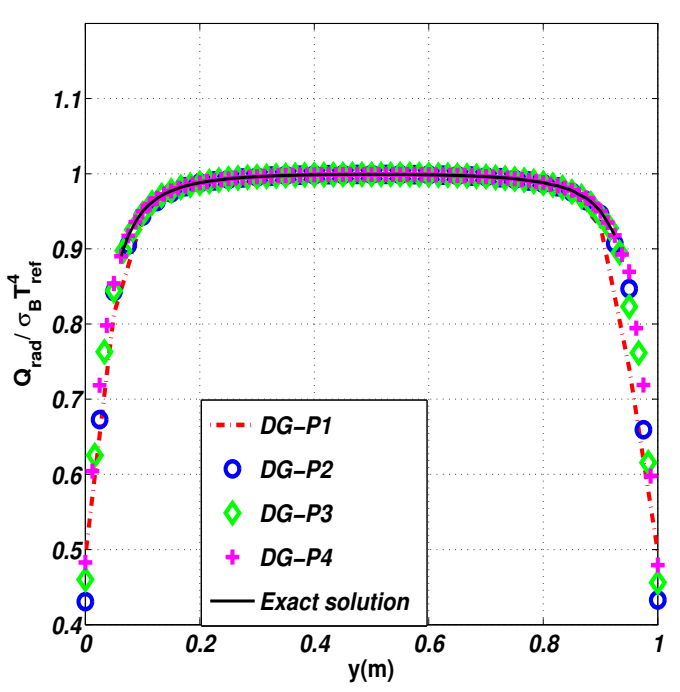

(b) $\kappa=10 m^{-1}$

Figure 4: Dimensionless radiative heat flux with the upwind DG method and, $S_{4}$

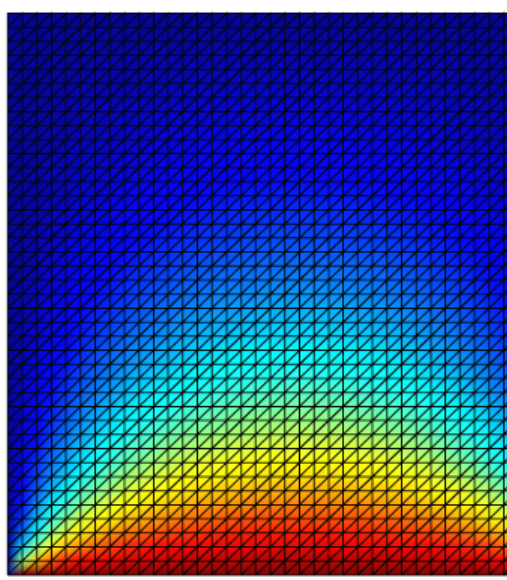

Temperature

0.75

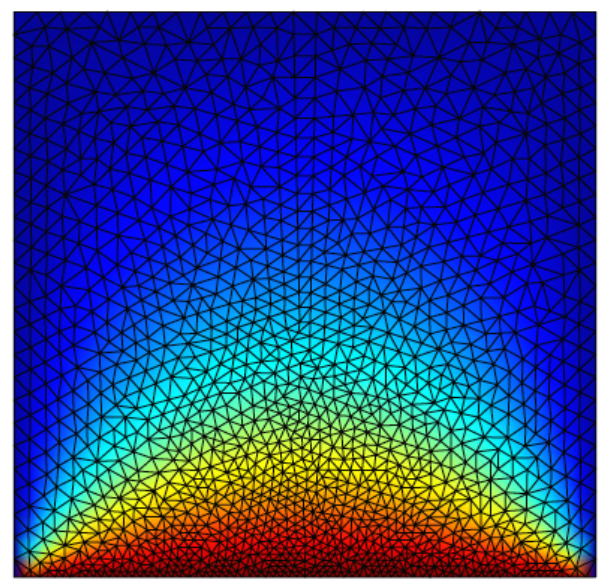

Temperature

0.75

(a)

(b)

Figure 5: The temperature distribution for $N_{s}=1$ for tow types of mesh grid. (a) a structured triangular grid composed of 1600 nodes $(h=0.025)$, (b) a refined mesh in hightemperature region composed of 1534 nodes. 


\subsection{1.a. Black surfaces.}

In this first test case, the medium boundaries were assumed to be black surfaces with imposed temperatures. This problem was investigated by Mishra et al. [22]. These authors used a Lattice Boltzmann method (LBM) to solve the energy equation and the collapsed-dimension method (CDM) was used to compute the radiative source term in the energy equation. This method was developed for 2-d simple geometries based on structured grids. Let us note that the same transient problem was also studied by Asllanaj et al. [20]. In this latter work, the RTE was solved by using a FVM based on a cell vertex scheme and associated to a modified exponential scheme. The PHAML code was used to solve the energy equation using finite elements method. The numerical solutions by Mishra et al. [22] were used as reference to check those obtained with our numerical method. Figure 6 and figure 7 show the evolution of the dimensionless temperature along the centerline position $s_{1}=0.5$ at different times and for different number $N_{s}=0.1$ and $N_{s}=1$. It should be noted that the computational time needed to achieve convergence of our algorithm increases with $N_{s}$. Obviously, more important the radiative part is, more quickly steady state was reached. Our results were in agreement with those of Asllanaj et al. [20] and Mishra et al. [32], confirming the validity of our numerical method for black surfaces.

Table 1: computational time in seconds to obtain a converged solution for $N_{s}=1$

\begin{tabular}{c|c|c|c|c}
\hline \multicolumn{5}{|c}{ EF-DG } \\
\hline$h$ & P1 & P2 & P3 & P4 \\
\hline 0.1 & 167.77 & 282.92 & 789.65 & $1.492 \times 10^{3}$ \\
& & & & \\
0.05 & 662.349 & $1.108 \times 10^{3}$ & $3.219 \times 10^{3}$ & $6.879 \times 10^{3}$ \\
& & & & \\
0.025 & $2.5135 \times 10^{3}$ & $5.183 \times 10^{3}$ & $8.783 \times 10^{3}$ & $12.43 \times 10^{3}$
\end{tabular}

Table 1 shows the computational time taken by our program to converge to the steady state solution using structured mesh $1, h=0.025$ correspond to the 1600 nodes and the other instead correspond to coarser mesh 1 grid. We tested the numerical stability for different temporal and spatial step discretization. This guarantees the stability of our combined numerical scheme. 


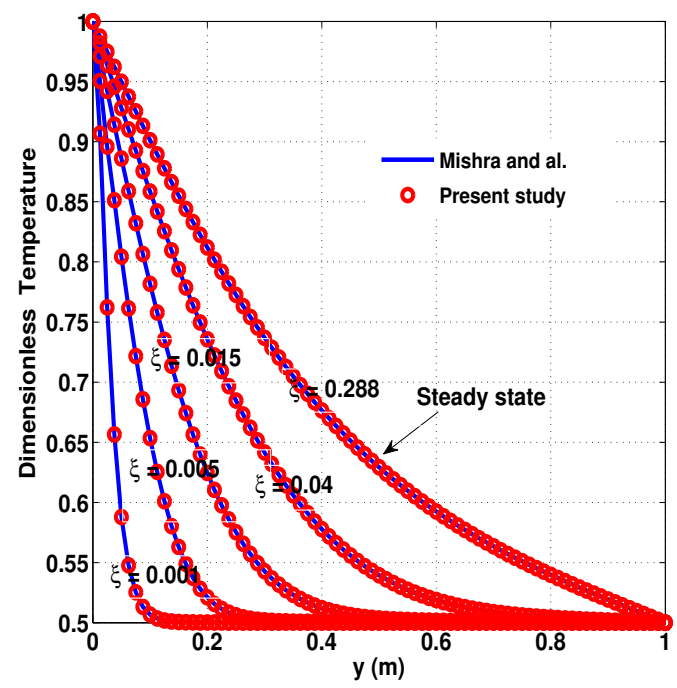

(a) $N_{s}=1$

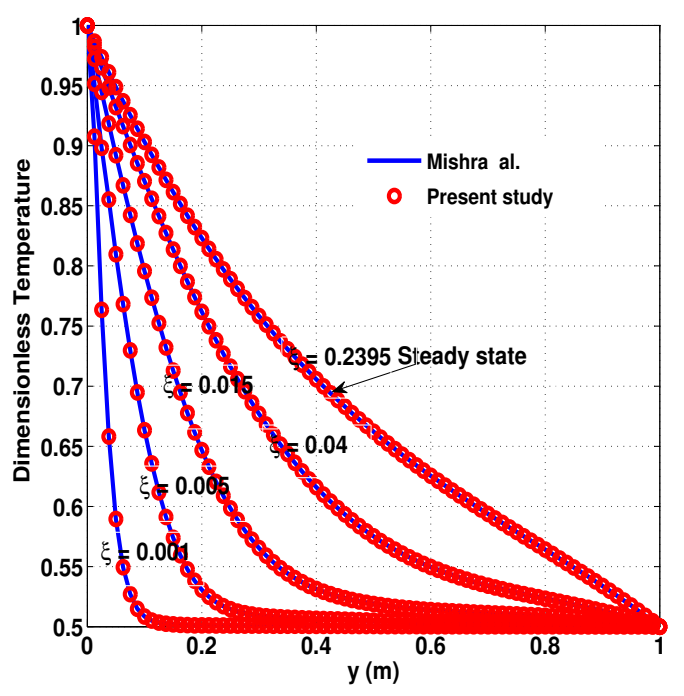

(b) $N_{s}=0.1$

Figure 6: Dimensionless temperature along the centerline position using mesh 1 at different dimensionless times and for two different values of the conduction-radiation parameter.

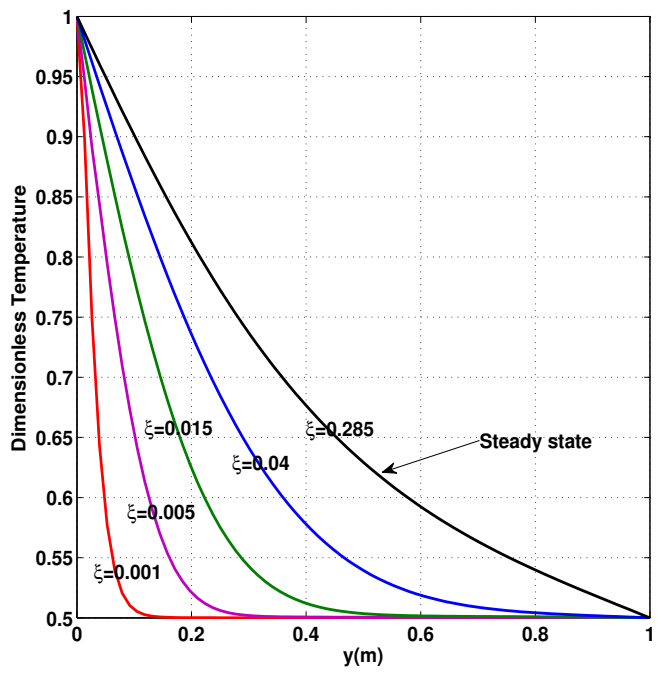

(a) $N_{s}=1$

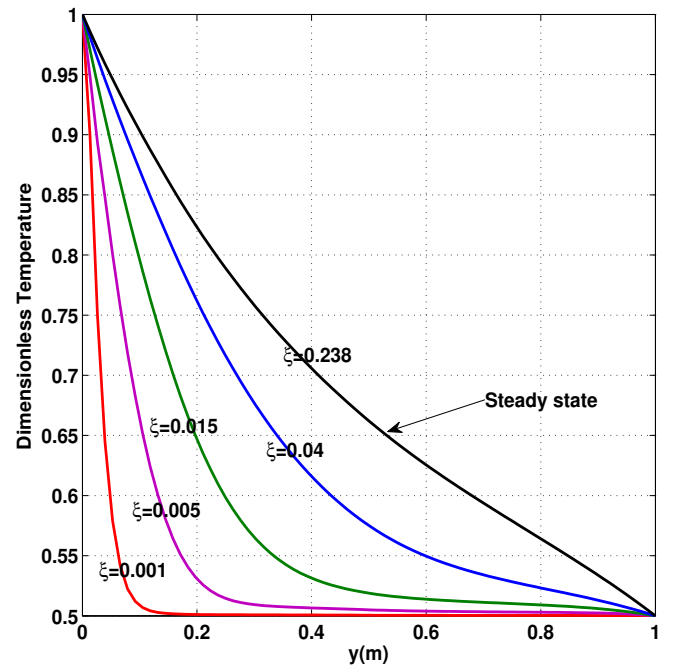

(b) $N_{s}=0.1$

Figure 7: Dimensionless temperature along the centerline position using mesh 2 at different dimensionless times and for two different values of the conduction-radiation parameter. 


\subsection{1.b. Combined black and diffuse reflection opaque surfaces.}

In this second test case, the hot (south) surface was assumed to be opaque with diffuse reflection and the other three surfaces were black. The number $N_{s}$ was set at 0.01 . Figure 8 and figure 9 show the dimensionless temperature distributions along the centerline position for two values of the wall emissivity $\epsilon$ equal to 0.5 and 0.1 respectively. These also show that the physical time required to obtain a converged solution decreases with $\epsilon$. A steady state was reached as dimensionless time $\xi=0.096$ for $\epsilon=0.5$ and $\xi=0.1070$ for $\epsilon=0.1$ when we use mesh 1 and as dimensionless time $\xi=0.096$ for $\epsilon=0.5$ and $\xi=0.1070$ for $\epsilon=0.1$ using mesh 2. Our results were found to comply with those of [32].

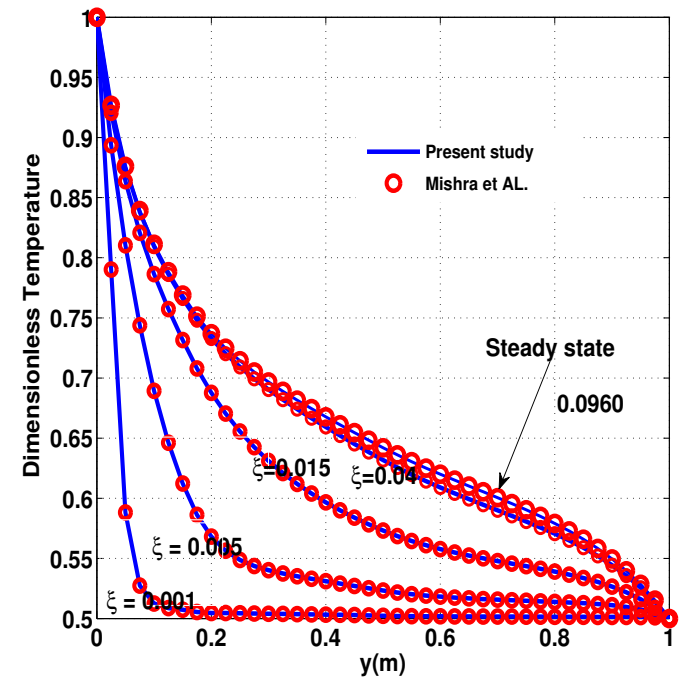

(a) $\epsilon=0.5$

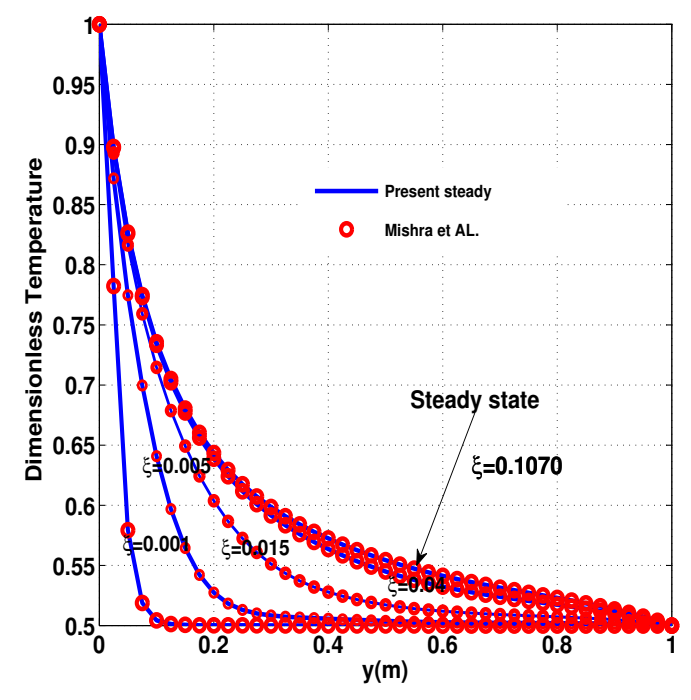

(b) $\epsilon=0.1$

Figure 8: Dimensionless temperature along the centerline position using mesh 1 at different dimensionless times and for two different values of the emissivity coefficient. 


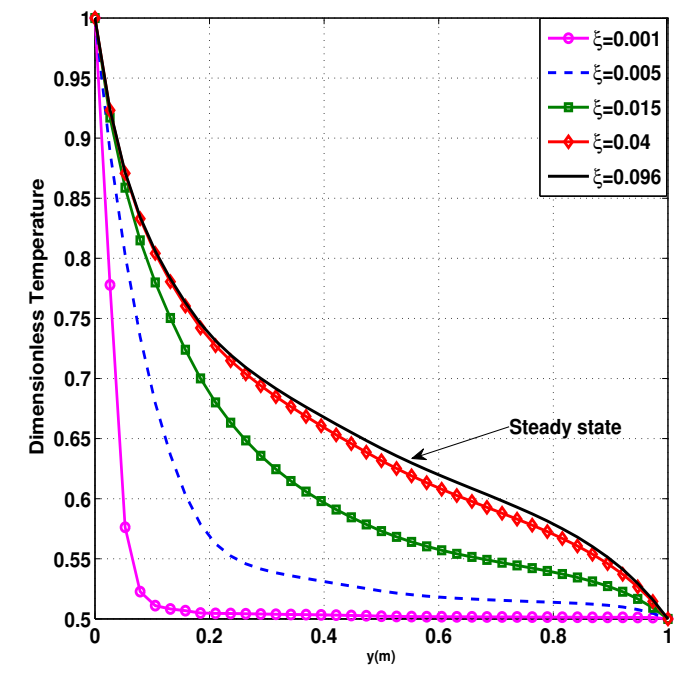

(a) $\epsilon=0.5$

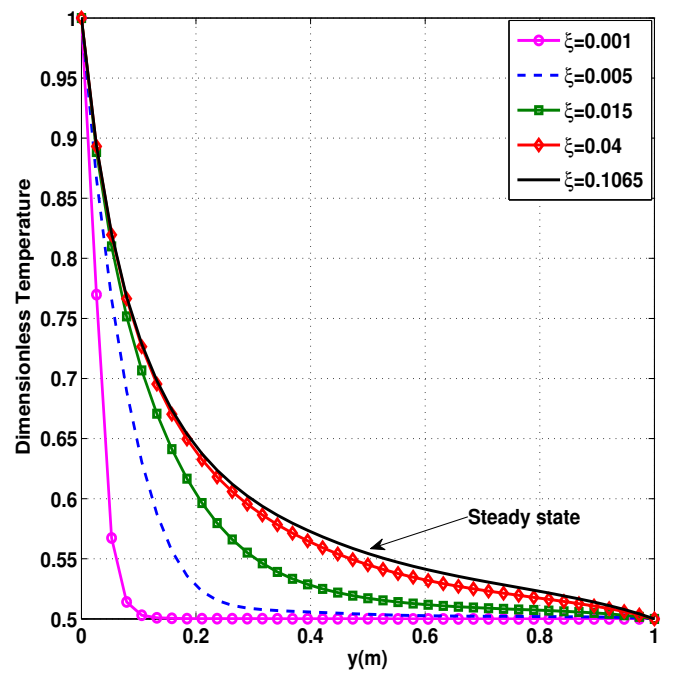

(b) $\epsilon=0.1$

Figure 9: Dimensionless temperature along the centerline position using mesh 2 at different dimensionless times and for two different values of the emissivity coefficient.

\subsection{1.c. Opaque surfaces with combined specular and diffuse reflec- tion}

The hot (south) surface was assumed to be opaque with specular reflection and the other three surfaces to be opaque with diffuse reflection. To our knowledge, this test case does not exist in literature in this area of research. For this instance we use a mesh 2. In figure 10 the dimensionless temperature is presented along the centerline position for three values of the wall emissivity $\epsilon(0.1,0.5$ and 0.9$)$ for all surfaces and with the conduction radiation number $N_{s}$ equal to 1 . The decrease in the emissivity coefficient was found to delay the steady state. The convergence was reached as $\xi=0.086$ for $\epsilon=0.9, \xi=0.1065$ for $\epsilon=0.5$ and $\xi=0.0998$ for $\epsilon=0.1$.

\subsubsection{Thermal Robin boundary conditions.}

In this section we considered the Robin boundary conditions (13). The medium boundaries were assumed to be black surfaces. To our knowledge, this is the first time this test case has been simulated. The simulation was 


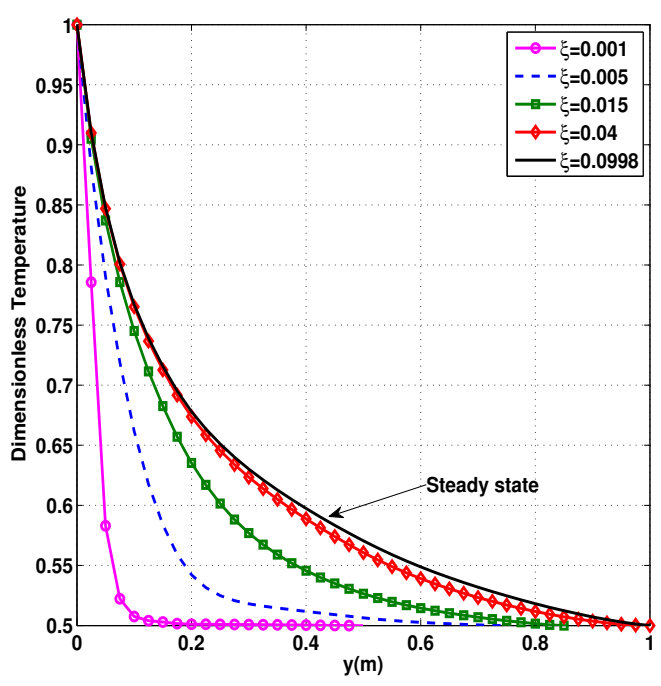

(a) $\epsilon=0.3$

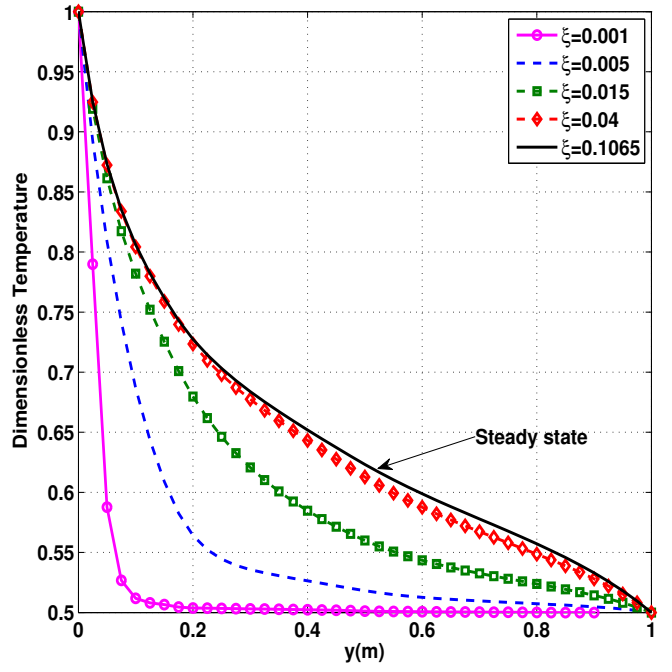

(b) $\epsilon=0.5$

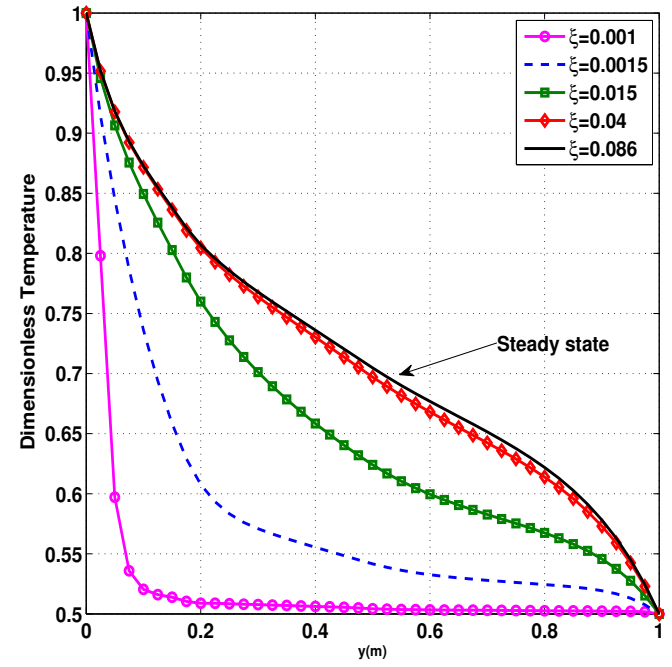

(c) $\epsilon=0.9$

Figure 10: Dimensionless temperature along the centerline position at different dimensionless times and for three different values of the emissivity coefficient. 
performed using a

$$
D_{1}=\left\{(x, y) \in \mathbf{R}^{2} ; 1 \leqslant x^{2}+y^{2} \leqslant 4,1 \leqslant|x| \leqslant 2 \text { and } 1 \leqslant|y| \leqslant 2\right\}
$$

and a refined mesh in high-temperature region composed of 1462 nodes. We assume the medium wall to be transparent (the gain by radiation and the radiative losses are neglected). Then

$$
-k_{c} \frac{\partial T^{\star}}{\partial n}=h_{c}\left(T^{\star}-T_{\infty}^{\star}\right)
$$

where $T_{\infty}^{\star}$ is the surrounding temperature and $h_{c}$ is the convective exchange coefficient of the wall. We introduce the Biot number

$$
B_{i}=\frac{h_{c} L}{k_{c}} .
$$

Now, we give to the dimensionless Robin boundary condition

$$
-\frac{\partial T}{\partial n}=B_{i}\left(T-T_{\infty}\right)
$$

In our numerical simulations, we take $B_{i}$ equal to 1 , this means that the heat conduction inside the body is lower than for the surface, see for instance [33]. The dimensionless surrounding temperature $T_{\infty}$ is equal to $1 \mathrm{in}$

$$
\Gamma_{1}=\left\{(x, y) \in \mathbf{R}^{2} ; x^{2}+y^{2}=1, y \leqslant 0\right\}
$$

and is equal to 0.5 elsewhere in the boundary. The figure 11 shows the field of the temperature for three values of the conduction radiation number $N_{s}$ equal to $1,0.1$ and 0.01 . Decreasing the conduction radiation number was found to also reduce the time of convergence to the steady state. The convergence was reached as dimensionless time $\xi=1.9090$ for $N_{s}=1, \xi=1.7225$ for $N_{s}=0.1$ and $\xi=1.6955$ for $N_{s}=0.01$.

\section{Conclusion.}

The aim of he work presented in this paper was to solve the combined radiation and conduction heat transfer in a grey absorbing and emitting medium. We used a high order DG method based on an upwind numerical flux to solve the radiative heat transfer problem. The DG method was 

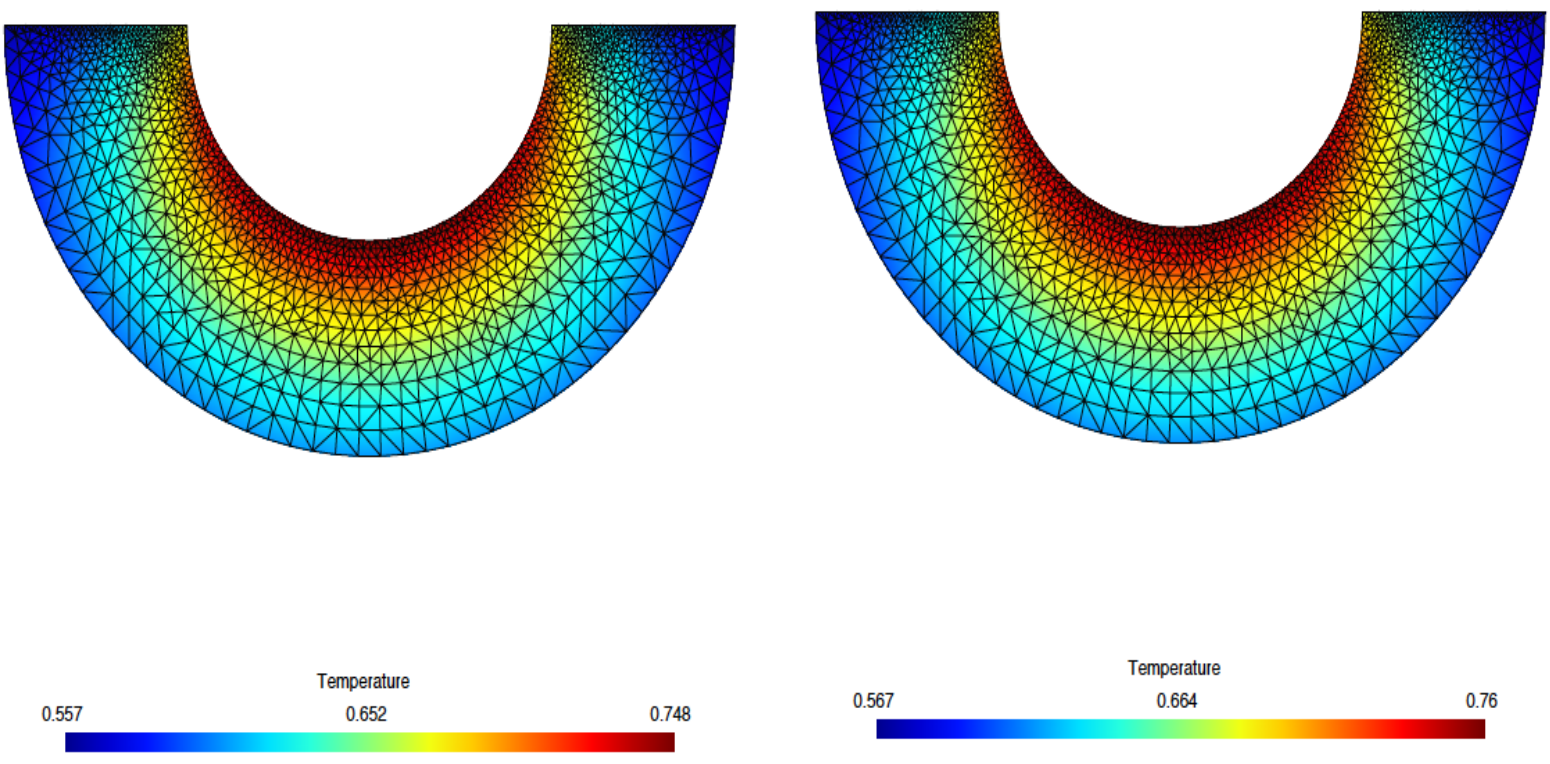

(a) $N_{s}=1$

(b) $N_{s}=0.1$
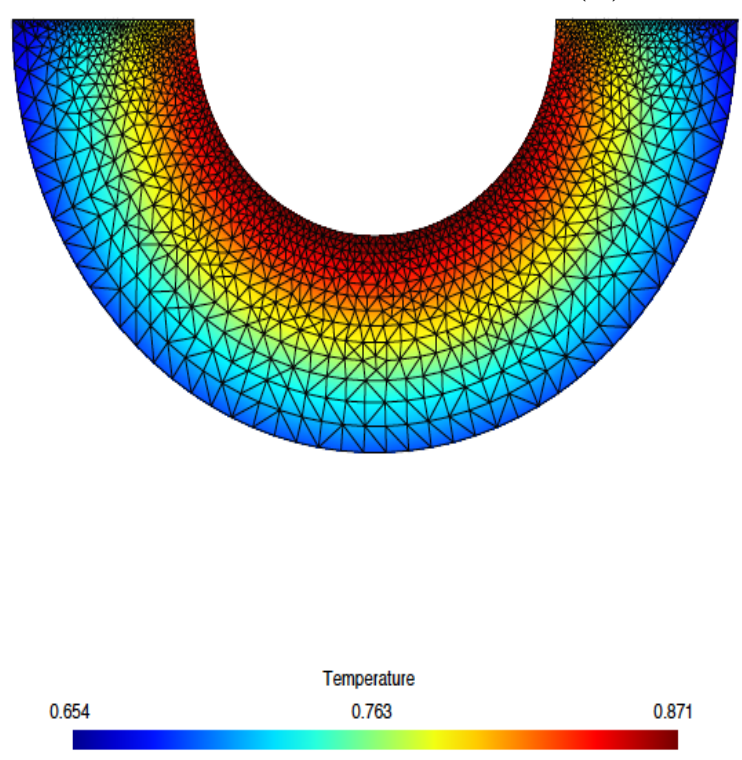

(c) $N_{s}=0.01$

Figure 11: The temperature distribution for different values of the conduction radiation number. 
coupled with the high order finite element method to solve the energy equation. Error estimates for the numerical solution were derived using suitable solution regularity assumptions in the RTE and we showed the stability of the DG method. Using the Newton method, a new treatment for a coupled nonlinear radiative-conductive system was implemented. We also investigated a new type of boundary conditions and showed that the numerical result was consistent with the theoretical findings. Three types of radiative boundary conditions were used -black walls, opaque walls with specular and diffuse reflection- and we explored the Dirichlet and Robin thermal boundary conditions.

The investigations of some basic aspects of the Continuous and Discontinuous Galerkin methods indicate that these methods are promising for solving the radiative-conductive heat transfer but much research still needs to be done in the future such that extending the idea to complex three dimensional geometries and an effective parallel implementation of the algorithms.

Acknowledgements. This work was partially supported by the French ANR-EMERGENCE and the pole MATERALIA.

\section{Appendix A. Dimensionless energy equation}

Let $\xi=\frac{\alpha t}{L^{2}}$ the dimensionless time where $\alpha=\frac{k_{c}}{\rho c_{p}}$ is the thermal diffusivity. We also introduce the conduction radiation number $N_{s}$ which satisfies the following expression:

$$
N_{s}=\frac{k_{c} \kappa}{4 \sigma_{B} T_{r e f}^{3}} .
$$

Let

$$
\begin{aligned}
& s=\frac{s \star}{L}, \tau=\frac{\alpha \tau^{\star}}{L^{2}}, T=\frac{T^{\star}}{T_{r e f}}, \\
& T_{0}(s)=\frac{T_{0}^{\star}(s)}{T_{r e f}}, G=\frac{G^{\star}}{4 \sigma_{B} T_{r e f}^{4}} \text { and } S_{r a d}=\frac{S_{r a d}^{\star}}{4 \sigma_{B} T_{r e f}^{4}} .
\end{aligned}
$$

Hence, the energy equation (10a)-(10b) can be written as the dimensionless form:

$$
\begin{array}{lc}
\frac{\partial T}{\partial \xi}-\triangle T+\theta T^{4}=\theta G, & \text { for } \quad(\xi, s) \in[0, \tau] \times \Omega, \\
T(0, s)=T_{0}(s), & \text { for all } s \in \Omega .
\end{array}
$$

where $\theta=\frac{\kappa^{2} L^{2}}{N_{s}}$ is the dimensionless constant. 


\section{Dimensionless radiative transfer equation} (2b):

Now, we give the dimensional form of radiative transfer equation (2a)-

$$
\begin{aligned}
\beta . \nabla I(\xi, s, \beta)+I(\xi, s, \beta) & =T^{4}(\xi, s), & & \text { for }(\xi, s, \beta) \in[0, \tau] \times \Omega \times \mathcal{D}, \\
I(\xi, s, \beta) & =g(\xi, s, \beta), & & \text { for } \quad(\xi, s, \beta) \in[0, \tau] \times \partial \Omega_{-} \times \mathcal{D},
\end{aligned}
$$

where

$$
I_{\text {ref }}=\frac{\sigma_{B}}{\pi} T_{r e f}^{4}, \quad I=\frac{I^{\star}}{I_{r e f}} \text { and } g=\frac{g^{\star}}{I_{\text {ref }}}
$$

are the dimensionless constant.

\section{References}

[1] F. Asllanaj, G. Jeandel, J. R. Roche, Numerical solution of radiative transfer equation coupled with nonlinear heat conduction equation, Internat. J. Numer. Methods Heat Fluid Flow. 11(5-6)(2001) 449-472.

[2] F. Asllanaj, A. Milandri, G. Jeandel, J. R. Roche, A finite difference solution of non-linear systems of radiative-conductive heat transfer equations, Internat. J. Numer. Methods Engrg. 54(11)(2002) 1649-1668.

[3] F. Asllanaj, G. Jeandel, J. R. Roche, Existence and uniqueness of a steady state solution of a coupled radiative-conductive heat transfer problem for a non-grey anisotropically and participating medium, Transport Theory Statist. Phys. 32(1)(2003) 1-35.

[4] F. Casenave, M. Ghattassi, R. Joubaud, A multiscale problem in thermal science.,ESAIM. 38 (2012) 202-219.

[5] F. Asllanaj, G. Jeandel, J. R. Roche, Convergence of a numerical scheme for a nonlinear coupled system of radiative-conductive heat transfer equations, Math. Models Methods Appl. Sci. 14(7)(2004) 943-974.

[6] F. Asllanaj, G. Jeandel, J. R. Roche, D. Lacroix, Transient combined radiation and conduction heat transfer in fibrous media with temperature and flux boundary conditions, International Journal of Thermal Sciences, 43 (2004) 939-950.

[7] J. R. Howell, The Monte Carlo Method in Radiative Heat Transfer, J. Heat Transfer 120(3) (1998) 547-560. 
[8] M. F. Modest, Radiative Heat Transfer, Academic Press, 2003.

[9] F. Asllanaj, V. Feldheim, P. Lybaert, Solution of Radiative Heat Transfer in 2-D Geometries by a Modified Finite-Volume Method Based on a Cell Vertex Scheme Using Unstructured Triangular Meshes, Numerical Heat Transfer, Part B: Fundamentals 51(2)(2007) 97-119.

[10] E. H. Chui, G. D. Raithby, computations of Radiant Heat Transfer on a Nonorthogonal Mesh Using the Finite-Volume Method ,Numerical Heat Transfer, Part B: Fundamentals 23(3)(1993) 269-288.

[11] P. Furmanski, J. Banaszek, Finite element analysis of concurrent radiation and conduction in participating media, Journal of Quantitative Spectroscopy and Radiative Transfer 84(4)(2004)563-573.

[12] B. Cockburn, C. W. Shu, Runge Kutta Discontinuous Galerkin Methods for Convection-Dominated Problems, J. Sci. Comput. 16(3)(2002)173261.

[13] J. S. Hesthaven, T. Warburton, Nodal Discontinuous Galerkin Methods, Springer, 2008.

[14] W. H. Reed, T. R. Hill, Triangular mesh methods for the neutron transport equation, (1973).

[15] P. Lesaint, P.A. Raviart, On a Finite Element Method for Solving the Neutron Transport Equation,Univ. Paris VI, Labo. Analyse Numrique, (1974).

[16] X. Cui, Q. Li Ben, A discontinuous finite-element formulation for multidimensional radiative transfer in absorbing, emitting, and scattering media, Numerical Heat Transfer, Part B: Fundamentals 46(5)(2004) 399428.

[17] X. Cui, Q. Li Ben, A Discontinuous Finite-Element Formulation for Radiative Transfer in Axisymmetric Finite Cylindrical Enclosures and Coupling with Other Mode Heat Transfer, Numerical Heat Transfer, Part B: Fundamentals 48(4)(2005) 317-344.

[18] F. Brezzi, B. Cockburn, L.D. Marini, E. Suli, Stabilization mechanisms in Discontinuous Galerkin finite element methods, Comput. Methods Appl. Mech. Engrg 195(25-28)(2006) 3293-3310. 
[19] F. Brezzi, L.D. Marini, E. Suli, Discontinuous Galerkin methods for first-order hyperbolic problems, Mathematical Models and Methods in Applied Sciences 14(2) (2004)1893-1903.

[20] F. Asllanaj, G. Parent, G. Jeandel, Transient Radiation and Conduction Heat Transfer in a Gray Absorbing-Emitting Medium Applied on TwoDimensional Complex-Shaped Domains, Numerical Heat Transfer, Part B: Fundamentals 52(2)(2007) 179-200.

[21] S.C. Mishra, H. Sahai, Analysis of non-Fourier conduction and radiation in a cylindrical medium using lattice Boltzmann method and finite volume method, International Journal of Heat and Mass Transfer 61(2013) $41-55$.

[22] S.C. Mishra, A. Lankadasu, Application of the lattice Boltzmann method for solving the energy equation of a 2-D transient conduction radiation problem, International Journal of Heat and Mass Transfer 48(17)(2005) 3648-3659.

[23] T. Cazenave, A. Haraux, An Introduction to Semilinear Evolution Equations, Clarendon Press Oxford, 1998.

[24] M. Zlamal, A finite element solution of the nonlinear heat equation, RAIRO Numer. Anal. 14 (1980) 203-216.

[25] K.Chrysafinos, L. S. Hou, Error estimates for semi-discrete finite element approximation of linear and semilinear parabolic equations under minimal regularity assumptions, SIAM journal on Numerical Analysis, 40(1)(2002) 282-306.

[26] P. G. Ciarlet, The Finite Element Method for Elliptic Problems, Society for Industrial and Applied Mathematics, 2002.

[27] D.A. Pietro, A. Ern, Mathematical Aspects of Discontinuous Galerkin Methods, Springer, 2011.

[28] W., Han, J. Huang, J. A. Eichholz, Discrete-Ordinate Discontinuous Galerkin Methods for Solving the Radiative Transfer Equation, SIAM J. Sci. Comput. 32(2)(2010) 477-497. 
[29] N. Shah, New method of computations of radiation heat transfer in combustion chambers, Ph.D. dissertation, Departement of Mechanical Engineering, 1979.

[30] W. A. Fiveland, Discrete-Ordinates Solutions of the Radiative Transport Equation for Rectangular Enclosures, J. Heat Transfer 106(4) (1984) 699-706.

[31] M. Asadzadeh, Analysis of a Fully Discrete Scheme for Neutron Transport in Two- Dimensional Geometry, SIAM Journal on Numerical Analysis 23(3) (1986), 543-561.

[32] S. C. Mishra, P. Talukdar, D. Trimis, F. Durst, computational efficiency improvements of the radiative transfer problems with or without conduction a comparison of the collapsed dimension method and the discrete transfer method, International Journal of Heat and Mass Transfer 46(16)(2003),3083-3095.

[33] K. D. Cole, J. V. Beck, K. A. Woodbury, F. Monte, Intrinsic verification and a heat conduction database, International Journal of Thermal Sciences 78 (2014),36-47. 